\title{
Philosophiques
}

\section{Représentation de groupe et démocratie délibérative : une alliance malaisée}

\section{Melissa S. Williams}

Volume 29, numéro 2, automne 2002

La démocratie délibérative

URI : https://id.erudit.org/iderudit/006252ar

DOI : https://doi.org/10.7202/006252ar

Aller au sommaire du numéro

\section{Éditeur(s)}

Société de philosophie du Québec

ISSN

0316-2923 (imprimé)

1492-1391 (numérique)

Découvrir la revue

Citer cet article

Williams, M. S. (2002). Représentation de groupe et démocratie délibérative : une alliance malaisée. Philosophiques, 29(2), 215-249.

https://doi.org/10.7202/006252ar
Résumé de l'article

Cet article examine la place du concept d'impartialité dans les théories délibératives de la démocratie. C'est à partir de certaines critiques féministes que sont discutés deux défis lancés à la théorie délibérative et qui sont étroitement liés : le premier porte essentiellement sur le critère du raisonnable et l'idée d'offre de raisons ; le second concerne les circonstances sociales et politiques contingentes dans lesquelles les perspectives des groupes

marginalisés peuvent influencer le jugement des autres citoyens. Certains des changements qui devraient être apportés à la théorie délibérative afin qu'elle puisse tenir compte de ces préoccupations sont ensuite proposés. Finalement, les implications de tels changements pour nos notions plus générales de vertu et de responsabilité de la citoyenneté sont examinées. 


\title{
Représentation de groupe et démocratie délibérative : une alliance malaisée ${ }^{1}$
}

\author{
MELISSA S. WILLIAMS \\ University of Toronto \\ mwilliams@chass.utoronto.ca
}

\begin{abstract}
RÉSUMÉ. - Cet article examine la place du concept d'impartialité dans les théories délibératives de la démocratie. C'est à partir de certaines critiques féministes que sont discutés deux défis lancés à la théorie délibérative et qui sont étroitement liés : le premier porte essentiellement sur le critère du raisonnable et l'idée d'offre de raisons; le second concerne les circonstances sociales et politiques contingentes dans lesquelles les perspectives des groupes marginalisés peuvent influencer le jugement des autres citoyens. Certains des changements qui devraient être apportés à la théorie délibérative afin qu'elle puisse tenir compte de ces préoccupations sont ensuite proposés. Finalement, les implications de tels changements pour nos notions plus générales de vertu et de responsabilité de la citoyenneté sont examinées.
\end{abstract}

\begin{abstract}
This article examines the place of the concept of impartiality in theories of deliberative democracy. Drawing on and extending recent feminist critiques of deliberative democracy, it explores two interrelated challenges to deliberative theory, one focused on the standard of reasonableness and the idea of reason-giving and the other on the contingent social and political circumstances under which marginalized-groups perspectives may sway the judgment of other citizens. Finally, some of the changes required of deliberative theory to address these concerns are discussed, and the implications of these changes for our more general notions of the virtues and responsibilities of citizenship are explored.
\end{abstract}

Ces dernières années, plusieurs théoriciens de la démocratie en sont venus à la conclusion que la justice démocratique envers les minorités ou les groupes désavantagés exige leur représentation adéquate dans les processus de décision politique ${ }^{2}$. Les politiques qui sont élaborées sans la participation active des membres de ces groupes risquent d'être biaisées ou incomplètes de diverses manières. Là où les relations d'inégalité sociale et politique sont structurées depuis longtemps en suivant les lignes de l'identité de groupe, il n'y a pas de fondement adéquat à la confiance entre les citoyens appartenant à des groupes marginalisés et les représentants qui sont membres de groupes privilégiés. Sans

1. Une version antérieure de cet article a été rédigée au cours de l'année pendant laquelle j'étais détentrice d'une bourse de recherche du « Program in Ethics and the Professions» à I'université $\mathrm{H}$ arvard. II fait partie d'un projet de recherche plus important pour lequel j'ai obtenu une subvention du Conseil de recherche en sciences humaines du Canada. Je remercie ces deux institutions pour leur soutien. J'aimerais également remercier Edward Andrew, Joseph Carens, David Kahane, Will Kymlicka, D ominique Leydet, Patchen $\mathrm{M}$ arkell, Wayne $\mathrm{N}$ orman, Alec Whalen et $D$ avid Welch pour leurs commentaires pertinents sur des versions préliminaires.

2. Voir en particulier Young, 1990, pp. 185-91; 1994; Guinier, 1994; Phillips, 1995; Williams, $1995 ; 1998$. 


\section{6 - Philosophiques / Automne 2002}

cette confiance, la communication, qui est une condition préalable à la représentation effective, a peu de chances d'exister. De plus, I'expérience qu'ont ces groupes de la marginalisation leur donne une compréhension des pratiques et des institutions sociales qui n'est pas immédiatement disponible aux individus n'ayant pas une telle expérience. L'appartenance à un groupe marginalisé implique souvent une voix politique distincte, et une représentation effective de tels groupes exige que cette voix se fasse entendre dans le processus politique.

M ême si certains groupes marginalisés sont effectivement représentés dans les processus de décision, leur seule présence peut n'avoir aucun effet sur les résultats de ces processus. Si la prise de décision est de type compétitif et majoritaire, rien n'empêche les plus puissants et les plus nombreux d'ignorer les voix des groupes marginalisés ${ }^{3}$. Si l'on suppose que les principes démocratiques de l'égalité individuelle interdisent la sur-représentation systématique des minorités dans les forums décisionnels, cela implique que la présence des groupes marginalisés ne pourra avoir d'impact durable sur les décisions politiques que si ces décisions sont fondées non seulement sur le décompte des votes, mais aussi sur le partage des raisons. Ainsi, la défense d'une représentation de groupe dépend d'une manière importante de la promotion des caractéristiques délibératives de la prise de décision politique - soit, celles qui visent l'entente fondée sur des principes - et du rejet du «renvoi d'ascenceur » (logrolling) ou des caractéristiques compétitives par lesquelles la faiblesse du nombre et des ressources limitées se traduisent en une incapacité d'obtenir des compromis des autres acteurs.

Ceci suggère une alliance naturelle entre défenseurs de la représentation de groupe et théoriciens de la démocratie délibérative. Le défi que lance le pluralisme à la légitimité politique est au coeur même du projet de la démocratie délibérative. Autant le pluralisme moral que la diversité sociale sapent la neutralité dont se réclame l'État libéral et exigent un autre fondement de la légitimité. Les démocrates délibératifs étendent l'idée libérale selon laquelle un gouvernement légitime est fondé sur le consentement, en montrant que les termes de la coopération sociale et politique devraient être le produit d'un échange raisonné entre citoyens. A fin de soutenir cette prétention à la légitimité, les processus de la démocratie délibérative doivent inclure toutes les perspectives sociales et politiques pertinentes. Tout comme les théoriciens de la différence ont voulu montrer que la justice envers les groupes marginalisés requiert des processus délibératifs de décision politique, les démocrates délibératifs ont soutenu qu'une description complète des processus délibératifs justes et légitimes doit porter attention à la place des besoins et des identités des groupes marginalisés dans l'échange discursif ${ }^{4}$. N ous retrouvons donc un lien entre représentation de groupe et démocratie délibérative dans ces deux courants de la théorie démocratique.

3. Pour une discussion de ce problème, voir Beitz, 1988, p. 135 ; Guinier, 1994, p. 105108, et Williams, 1998, chapitres 4 et 7.

4. Voir par ex., Sunstein, 1988; H abermas, 1996a, p. 183. 
M algré ces affinités importantes entre les arguments en faveur de la représentation de groupe et de la démocratie délibérative, ceux-ci ne sont pas coextensifs et il existe également d'importantes tensions entre eux. Certaines de ces tensions ont été rel evées dans de récentes discussions féministes de la théorie délibérative auxquelles les démocrates délibératifs ont répliqué. $M$ algré ma profonde sympathie pour le projet de la démocratie délibérative, je ne suis pas persuadée que ses défenseurs ont relevé de façon adéquate les défis que pose la différence sociale - différence selon le sexe, la race, I'ethnie, la classe, la sexualité, etc. - envers la conception délibérative de la légitimité. En effet, si nous prenons au sérieux les défis de la différence sociale, nous découvrons qu'ils menacent d'affaiblir les notions mêmes du raisonnable et de l'offre de raisons sur lesquelles la théorie délibérative dépend pour sa conception de la légitimité. Plus particulièrement, nous découvrons que le fait que les citoyens reconnaissent ou non les raisons d'autrui comme raisons pourrait s'avérer un phénomène socio-culturel contingent. De plus, il semble probable que la contingence de cette reconnaissance aura tendance à désavantager systématiquement les raisons des groupes marginalisés dans un échange discursif. Bien que la théorie de la démocratie délibérative soit sans doute la meilleure description de la légitimité dont nous disposions, elle demeure incomplète tant qu'elle ne confronte pas ce problème.

Les théoriciens de la différence de groupe ont été très critiques des revendications de neutralité et d'impartialité de l'État libéral : les revendications d'impartialité, précisent-ils, se paient toujours par la suppression des différences sociales. Une des visées centrales de la théorie délibérative est de réhabiliter l'idéal d'impartialité en élaborant des processus politiques de manière à éviter tout préjugé envers des intérêts sociaux valables. La prochaine section de cette étude présente dans leurs grandes lignes des théories de la démocratie délibérative et explore la place du concept d'impartialité dans ces théories ${ }^{5}$. $\mathrm{N}$ ous verrons que pour les démocrates délibératifs, l'impartialité représente une vertu autant des institutions et des procédures que des citoyens individuels. J'explore dans la seconde section les différents types de contributions que les perspectives des groupes marginalisés apportent à la délibération démocratique. $D$ ans la troisième section, je $m$ 'inspire des récentes critiques féministes de la démocratie délibérative afin de mettre en évidence deux défis lancés à la théorie délibérative et qui sont étroitement liés : le premier porte essentiellement sur le critère du raisonnable et l'idée d'offre de raisons; le second concerne les circonstances sociales et politiques contingentes dans lesquelles les perspectives des groupes marginalisés peuvent influencer le jugement des autres citoyens. Finalement, je propose certains des changements qui devraient être apportés à la théorie délibérative afin qu'elle puisse tenir compte de ces préoccupations, et j'examine les implications de tels changements pour

5. Cette discussion relie cette étude à un projet plus important, dont le titre provisoire est Reconstructing I mpartiality, dont elle fera partie. 


\section{8 - Philosophiques / Automne 2002}

nos notions plus générales de vertu et de responsabilité de la citoyenneté. Cette étude comprend aussi un appendice qui présente en résumé un débat au Sénat des États-Unis illustrant la contingence de l'offre de raisons dans la discussion publique.

\section{La démocratie délibérative et l'idéal d'impartialité}

Les théories de la démocratie délibérative s'appuient sur l'idéal d'impartialité comme principe régulateur, de manières diverses et de façon plus ou moins explicite $^{6}$. Avant de passer à la discussion de la place de l'impartialité dans ces théories, il serait utile de présenter une brève esquisse de ce qu'est la démocratie délibérative ${ }^{7}$. Les théories de la démocratie délibérative s'intéressent, d'abord et avant tout, au problème de la justification politique dans le contexte du désaccord moral, problème qu'elles ont pour but de résoudre. $D$ 'après l'idéal de la démocratie délibérative, nous dit Joshua Cohen, «la justification de l'exercice du pouvoir politique collectif doit procéder sur la base d'un échange de raisons libre et public entre égaux $\gg^{8}$. Toutes les formes de discussion politique ne se conforment pas à cet idéal de la démocratie délibérative; l'idée du débat libre et raisonnable entre égaux sert de contrainte autant au contexte qu'au contenu de la discussion. Plus important encore, la démocratie délibé rative requiert que le contexte de la discussion politique soit tel que les citoyens qui y participent entretiennent une relation mutuelle d'égalité et recherchent l'accord moral sur les aspects importants de leur vie sociale et politique partagée. L'idée au coeur de la démocratie délibérative, selon Gutmann et Thompson, est le principe de réciprocité qui « requiert des citoyens qu'ils persistent à chercher des termes équitables de coopération entre égaux $» 9$.

En ce qui concerne le contenu de la discussion politique, les théories de la démocratie délibérative imposent quelques contraintes supplémentaires importantes. Les participants à la délibération démocratique doivent défendre la conception de l'intérêt public ou du bien commun qu'ils favorisent sur la base de raisons morales ou éthiques acceptables pour tous les participants. Cette

6. Ainsi, l'un des deux piliers de la théorie de l'éthique de la discussion de $\mathrm{H}$ abermas, le principe de discussion, «n'élucide que le point de vue selon lequel les normes d'action en général peuvent être fondées en raison de manière impartiale» (H abermas, 1997, p. 125; italiques originales). Le principe de discussion stipule que : «Sont valides strictement les normes d'action sur lesquelles toutes les personnes susceptibles d'être concernées d'une façon ou d'une autre pourraient se mettre d'accord en tant que participants à des discussions rationnelles» (I bid., p. 123). Voir également Sunstein, 1993, p. 17.

7. Dans cette discussion, j'inclus un vaste éventail de théoriciens contemporains dans la catégorie de « démocrate délibératif ». II existe des divergences évidentes entre ces théoriciens, particulièrement selon qu'ils ont été influencés davantage par les travaux de John Rawls ou de Jürgen $\mathrm{H}$ abermas. Toutefois, pour les thèmes qui me concernent le plus ici, les similitudes entre ces théoriciens sont plus pertinentes que leurs différences.

8. Cohen, 1996, p. 99; voir aussi Cohen, 1989; M anin, 1987.

9. Gutmann et Thompson, 1996, p. 53. Toutes les traductions en français sont de nous, sauf lorsqu'indiqué. (N.d.T.) 
conception de la légitimité démocratique se distingue notamment des conceptions de la politique fondées sur les stratégies d'avantage mutuel ${ }^{10}$. La légitimité de l'action politique dépend non pas du fait qu'elle sert d'une manière égale les intérêts de tous les citoyens, mais plutôt de ce qu'elle soit fondée sur des raisons que tous considèrent comme valides. En quoi consistent des raisons mutuellement acceptables? Elles ne doivent surtout pas référer à des prémisses qui font l'objet d'un désaccord raisonnable et fondamental; autrement dit, des prémisses dont la plausibilité ne peut être établie par des «modes d'enquête rela-

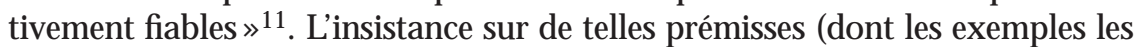
plus limpides proviennent des commandements de la foi religieuse révélée) anéantit l'égalité du rapport entre les participants à la discussion en privilégiant les engagements moraux de certains au détriment des autres ${ }^{12}$.

Au niveau phénoménologique, la démocratie délibérative ressemble à peu près à ceci : nous offrons les uns aux autres des arguments sur les exigences du bien commun et nous tentons de nous convaincre les uns les autres de notre propre compréhension de ce bien. $\mathrm{N}$ os arguments se fondent sur des raisons que nous croyons acceptables pour les autres, et ils visent ce qui est commun plutôt que ce qui est particulier à un individu ou à un groupe. Ainsi, nous ne faisons pas appel à l'autorité divine ou à des conceptions controversées de la nature humaine comme fondement ultime de ce que nous demandons des autres citoyens. Les exigences que nous formulons envers des individus ou des groupes doivent être structurées non pas en terme de leurs intérêts étroits, mais en terme de nos engagements partagés envers des fins publiques, incluant celle de la justice. En nous exprimant, nous assumons donc notre identité de citoyens intéressés par le bien commun, et non pas celle de porteurs d'intérêts particuliers (sauf dans la mesure où ils éclairent nos fins partagées). Finalement, les exigences de la démocratie délibérative concernent autant notre caractère que la façon dont nous nous projetons en public : nous nous devons d'engager la conversation avec une ouverture d'esprit suffisante pour que nous puissions être persuadés par les arguments d'autrui.

Comment une telle persuasion se produit-elle? N ous découvrons des manières de caractériser des questions d'intérêt commun que nous ne connaissions pas et qui nous les font envisager sous un jour différent : elles rendent mieux compte des faits en cause et de nos propres intuitions morales conflictuelles que les interprétations que nous avions jusque là affirmées ${ }^{13}$. Par ce procédé, nous réduisons la portée du désaccord, et nos décisions collectives reflètent un plus grand degré d'un sens commun de la question que nous ne

10. Pour des critiques des conceptions de la justice fondées sur l'avantage mutuel, voir Barry, 1995, chapitre 2 et Gutmann et Thompson, 1996, p. 57-58.

11. Gutmann et Thompson, 1996, p. 56.

12. Voir Cohen, 1996, p. 101.

13. Comme le suggère cette formulation, il me semble probable que la phénoménologie de la persuasion de la démocratie délibérative possède d'importantes similitudes avec l'idée de l'équilibre réfléchi dont dépend si fortement la conception de la justification de Rawls. 


\section{0 - Philosophiques / Automne 2002}

possédions avant la discussion. Dans la mesure où aucune voix n'a été réduite au silence au cours du processus délibératif et que les arguments de chacun ont été écoutés avec ouverture d'esprit, les décisions auxquelles nous aboutissons ont une plus grande légitimité que celle qu'elles auraient pu obtenir par toute autre méthode de décision.

R endre compte des façons dont l'idéal d'impartialité est impliqué par les théories de la démocratie délibérative est une tâche qui se complique par la multiplicité des significations du terme «impartialité». En effet, certains théoriciens évitent tout simplement ce terme parce qu'il est étroitement associé aux notions de raisonnement déductif, de l'idéal du juge impersonnel et d'intenables prétentions à l'autorité morale - bref, des éléments caractéristiques de ce quej'ai nommé le modèle juridique de la justice ${ }^{14}$. M ais ces notions n'épuisent pas toutes les manières de penser l'impartialité, et plusieurs autres conceptions de l'impartialité imprègnent l'ensemble des discussions en démocratie délibérative. La signification de l'impartialité comme un élément essentiel (sinon le seul) de la justice dans une société démocratique est particulièrement éclairée par l'examen de la double signification de son contraire, la partialité. La justice d'un jugement ou d'une politique dépend, d'abord, de ce qu'il n'est pas partiel au sens $d^{\prime}$ « incomplet» ${ }^{15}$ : il doit considérer toute la preuve, toutes les perspectives et toutes les personnes. Ensuite, la justice dépend de jugements et de politiques qui ne sont pas partiaux au sens de «biaisé» : ils ne doivent pas favoriser certains au détriment des autres sur des bases moralement arbitraires. Comme le dit Benhabib, "Ce qui est considéré impartial doit être dans "les meilleurs intérêts de tous égal ement" ${ }^{16}$. D e plus, I'impartialité ainsi comprise peut être une vertu des individus délibérant, une vertu des institutions et des pratiques ou une vertu des lois et des politiques.

Le principe d'impartialité imprègne les caractérisations de l'idéal de la démocratie délibérative d'au moins six façons :

1. Impartialité comme inclusion : personnes. Toutes les théories de la démocratie délibérative font du caractère inclusif des processus démocratiques un élément central de leurs idéaux normatifs. Les décisions démocratiques doivent être fondées en principe sur la délibération de tous ${ }^{17}$.

14. Williams, 1995. Voir particulièrement la distinction de Gutmann et Thompson entre les principes d'impartialité et de réciprocité, ainsi que leur rejet du premier en faveur du second (1996, pp. 53-54, 59-63). Simultanément, ils reconnaissent d'emblée que ce qu'ils entendent par « réciprocité» ressemble beaucoup à la façon dont Brian Barry définit la «justice comme impartialité», soit «des principes et des règles qui sont susceptibles de former la base d'un accord libre entre individus qui recherchent un accord selon des termes raisonnables» (Barry, 1995, p. 11); voir Gutmann et Thompson, 1996, p. 373-74, notes 1-3). Cf. Sunstein, 1993, p. 24 : «le principe d'impartialité requiert que le gouvernement donne des raisons pouvant être intelligibles pour des gens différents qui opèrent sur la base de prémisses différentes».

15. L'auteur joue ici sur le fait que le terme «partial» en anglais recouvre les adjectifs «partial » et «partiel» en français. (N.d.T.)

16. Benhabib, 1996, p. 83; voir aussi Chambers, 1996, p. 100-1.

17. M anin, 1987, p. 352. 
John Dryzek exprime cette idéal ainsi : «Aucun individu concerné ne devrait être exclu et, si nécessaire, des mécanismes éducatifs devraient promouvoir la participation compétente des personnes possédant un intérêt matériel dans les questions en cause et qui, sans cela, pourraient être laissées pour compte» ${ }^{18}$. II est important de noter que l'inclusion comme élément de l'impartialité est substantiellement reliée à l'absence de parti-pris comme essence de l'impartialité. Comme le remarque Jürgen $\mathrm{H}$ abermas, « la participation effective de chaque personne concernée est seule à pouvoir prévenir les déformations de perspectives qu'introduit I'interprétation d'intérêts chaque fois personnels ${ }^{19}$. Q uoi qu'il en soit, la démocratie délibérative aspire au développement d'une communauté politique dans laquelle les décisions publiques sont justifiables à tous les citoyens, et où tous les acteurs publics considèrent qu'il est de leur responsabilité de défendre leurs actions par des raisons publiquement acceptables. Bien qu'il puisse y avoir certaines limites à notre capacité de réaliser cette aspiration, même en théorie, celle-ci demeure la fin régulatrice de la démocratie délibérative ${ }^{20}$.

2. Impartialité comme inclusion : questions. Les démocrates délibératifs cherchent à structurer les institutions et les processus politiques de manière à ce que toutes les considérations pertinentes soient prises en compte dans les décisions politiques. La quête de l'inclusion s'opère à deux niveaux. D'abord, la démocratie délibérative vise à s'assurer que toutes les raisons et les éléments pertinents à une décision particulière aient un poid dans le processus délibératif ${ }^{21}$. Ensuite, aucun sujet $n^{\prime}$ 'st en principe à l'abri de la discussion publique. Contrairement aux libéraux rawlsiens, les démocrates délibératifs ne voudraient pas même retirer les droits et libertés fondamentaux de l'ordre du jour politique ${ }^{22}$. L'ouverture de cet ordre du jour signifie que les questions précédemment

18. Dryzek, 1990, p. 43.

19. H abermas, 1986, p. 89.

20. Voir notamment Gutmann et Thompson, 1996, p. 128, où ils considèrent les difficultés que pose l'accomplissement d'une telle responsabilité universelle, autant au niveau théorique que pratique. Voir aussi Chambers, 1996, p. 197-98.

21. Voir par ex. M anin, 1987; cf. Cohen et Rogers, 1995, pp. 42-43. Comme j'aurai l'occasion de le montrer plus bas, l'inclusion par rapport aux questions est importante car la qualité (qui se distingue de la justice) des décisions publiques dépend d'une considération de tous les arguments pertinents. En partie, pourtant, cette forme d'inclusion est un ajout important à l'inclusion de toutes les personnes pertinentes au processus délibératif dans les cas où l'inclusion des personnes ne garantit pas automatiquement l'inclusion des raisons. Cela se voit particulièrement dans les domaines où les citoyens ne peuvent pas défendre leurs propres intérêts adéquatement, comme c'est souvent le cas avec les enfants et les handicapés mentaux.

22. Voir notamment Benhabib, 1996, p. 79-80 où elle soutient que le contenu des droits fondamentaux humains et civils se voit toujours essentiellement contesté en démocratie. Benhabib formule cette proposition comme un compte rendu empirique (correct selon moi) de la contestation des principes fondamentaux, mais également comme un élément de la théorie normative 
discutées peuvent toujours, potentiellement, faire l'objet d'un débat public ${ }^{23}$.

3. Impartialité comme absence de parti-pris : structure institutionnelle. La structure de la situation délibérative se veut elle-même impartiale, dans la mesure où ses participants sont caractérisés par une relation mutuelle de parfaite égalité. Aucun ne jouit d'un avantage structurel lui permettant de se faire entendre favorablement des autres; tout dépend du caractère persuasif des arguments eux-mêmes : «la participation à ... la délibération est gouvernée par des normes d'égalité et de symétrie; tous ont les mêmes chances d'amorcer des actes de langage, de questionner, d'interroger et d'ouvrir le débat » ${ }^{24}$. Bref, les relations de pouvoir n'ont pas leur place dans le processus délibératif.

4. Impartialité comme absence de parti-pris : raisons. Le principe au cœur de la démocratie délibérative est que les raisons qui sont utilisées afin de justifier les décisions politiques sont acceptables à tous. A insi, les raisons offertes par les participants pour soutenir leurs interprétations des fins partagées sont elles-mêmes des raisons «impartiales» en deux sens. D'abord, comme nous l'avons discuté plus haut, les raisons ne doivent pas s'appuyer sur des prémisses ne pouvant être affirmées que par quelques participants, les prémisses fondées sur une foi révélée en constituant l'exemple le plus net. Ensuite, les raisons devraient en appeler aux intérêts partagés par tous les participants plutôt qu'aux intérêts partiaux d'un sous-groupe quel conque. Lors d'un processus délibératif, l'argument voulant que ce qui est bon pour General M otors soit bon pour la nation, sans autre précision sur la façon dont les autres acteurs sociaux profiteraient de la prospérité de General M otors, n'a simplement aucune force persuasive. Finalement, l'impartialité des raisons devrait agir comme une contrainte à la présentation publique des décisions délibératives, de même qu'à leur développement. Comme l'explique Seyla Benhabib, «un idéal moral d'impartialité est un principe régulateur qui devrait gouverner non seulement nos délibérations en public, mais aussi I'articulation des raisons par les institutions publiques ${ }^{25}$.

5. Impartialité comme absence de parti-pris : participants. L'impartialité de la démocratie délibérative dépend de façon importante non seulement de la structure des institutions et du contenu de la discussion politique, mais également du caractère des citoyens démocratiques; c'est une vertu

de la démocratie délibérative. Comme Benhabib prend soin de le souligner, cela ne revient évidemment pas à dire que la démocratie délibérative permettrait à une telle contestation de rendre les droits fondamentaux susceptibles de changement par de simples procédures majoritaires. L'idée est que la démocratie délibérative ne cherche pas à soustraire quelque principe que ce soit de la critique et de la révision raisonnables.

23. Voir par ex. Gutmann et Thompson, 1996, p. 83.

24. Benhabib, 1996, p. 70.

25. Benhabib, 1996, p. 83. 
autant des individus que des institutions et des pratiques. Les participants devraient donner une «considération pleine et impartiale» aux divers principes et moyens d'action qui se présentent à eux ${ }^{26}$. Ainsi, l'impartialité est une caractéristique non seulement de l'esprit dans lequel chaque participant devrait parler, mais aussi de l'esprit dans lequel chacun devrait écouter et juger les paroles des autres ${ }^{27}$. Plus important encore, peut-être, la démocratie délibérative dépend de ce que les citoyens possèdent des qualités de caractère qui incluent l'ouverture d'esprit ou une volonté d'être persuadé par des raisons différentes de celles qu'on a proposées dans le forum public :

Des citoyens ouverts tentent de rompre des habitudes personnelles et institutionnelles qui les décourageraient d'accepter dans l'avenir des positions opposées ou, au moins, de modifier leur position en ce sens. Tant l'esprit politique que le forum politique devraient rester ouverts à la reconsidération de décisions déjà prises et de politiques déjà adoptées ${ }^{28}$.

6. Impartialité comme absence de parti-pris : résultats. La démocratie délibérative adopte comme prémisse et comme conclusion que les décisions politiques ne devraient pas favoriser systématiquement certaines personnes ou intérêts au détriment des autres. Cela ne revient pas à affirmer que les décisions délibératives n'exigeront pas parfois une distribution inégale de bénéfices ou de tâches, mais que chaque fois que de telles inégalités existent, elles seront justifiables par des raisons que tous pourraient accepter.

Je considère cet idéal de démocratie très séduisant, et je me situe parmi ceux qui affirment qu'un modèle de démocratie délibérative quel qu'il soit constitue un préalable nécessaire à toute défense cohérente de la représentation des groupes marginalisés ${ }^{29}$. Toutefois, je conserve, pour certaines raisons, des réserves quant à la capacité des versions existantes de la démocratie délibérative d'être des modèles de justice envers les groupes marginal isés. Le reste de cette étude va poursuivre l'examen de ces réserves que j'entretiens sur la capacité de la démocratie délibérative à servir les fins de la justice envers les groupes marginalisés. N ous verrons de quelles ressources nous disposons afin de les écarter. $\mathrm{N}$ ous procéderons en posant une question générale : comment une attention aux perspectives des groupes marginalisés peut-elle contribuer à la légitimité des décisions politiques qui sont le produit de la délibération démocratique?

26. Cohen et Rogers, 1995, p. 96.

27. Voir Williams, 1995, p. 85-87.

28. Gutmann et Thompson, 1996, p. 82-83.

29. Williams, 1998. 


\section{Les perspectives des groupes marginalisés en délibération démocratique}

Une pluralité de perspectives sociales est un présupposé nécessaire à la démocratie délibérative : sans elle il n'y aurait rien à gagner de la discussion ${ }^{30}$. Le pluralisme enrichit la délibération car il accroît le nombre de conceptions différentes d'un problème que nous pouvons considérer lorsque nous tentons de le résoudre. En particulier, la présence d'une pluralité de points de vue ou d'opinions dans la discussion politique améliore, sans doute, nos jugements et nos décisions en nous permettant d'anticiper certaines faiblesses et certains pièges associés à toute perspective particulière. Elle permet aux décisions d'être plus complètes, d'inclure un plus grand éventail de possibilités sociales et politiques et de prévoir un plus grand nombre de conséquences sociales et politiques de nos décisions.

Jusqu'ici il apparaît clairement que plusieurs formes de diversité sociale amélioreront le processus délibératif, et que les perspectives des groupes marginalisés constituent une dimension du pluralisme qui contribuera au caractère inclusif des décisions politiques au moins autant que toute autre dimension. En fait, il y a de bonnes raisons de supposer qu'elles y contribueront davantage, car historiquement ces perspectives ont eu tendance à être ignorées dans le processus politique. Les groupes marginalisés peuvent discerner des aspects des forces sociales qui ne sont pas évidents aux autres, et ce qu'ils perçoivent pourrait s'avérer extrêmement utile à l'élaboration d'une bonne politique publique ${ }^{31}$. Cette idée a soustendu certains des arguments les plus forts en faveur de l'extension du droit de vote aux femmes dans le mouvement suffragiste américain du début du siècle. Certaines militantes, notamment Jane Addams, ont voulu montrer que les transformations de la production industrielle ainsi que les dynamiques de l'urbanisation croissante faisaient en sorte que les fonctions sociales habituellement accomplies au sein des ménages (hygiène, traitement des déchets, santé) devaient maintenant être considérées comme des questions de politique publique. Parce que les femmes, selon Addams, étaient les agents qui, dans les ménages, avaient assumé ces rôles sociaux, elles possédaient une connaissance de ces besoins dont les hommes étaient généralement dépourvus. Les femmes devaient donc avoir une place égale en politique car l'élaboration d'une bonne politique publique dépendait $d^{\prime}$ une manière cruciale de leur contribution au processus décisionnel ${ }^{32}$.

M ême si la description précédente rend bien compte de la façon dont une politique délibérative plus inclusive sert notre intérêt dans des jugements politiques solides, cela toutefois ne nous dit pas grand chose de la façon dont une politique délibérative de la différence peut servir le but de la justice. En

30. Cf. Phillips, 1995, p. 151 : «La délibération n'a d'importance que parce qu'il y a différence; si les hasards de la nature ou de l'histoire avaient permis le développement d'une communauté politique fondée sur l'accord unanime, alors la politique serait virtuellement redondante et les décisions seraient déjà prises».

31. Voir Cohen et Rogers, 1995, pp. 42-43; Young, 1997b.

32. Addams, 1914. 
d'autres termes, si cette description nous offre bien des raisons qui nous montrent en quoi les perspectives des groupes marginalisés pourraient améliorer les politiques sociales, elle ne nous explique pas comment l'inclusion de ces perspectives dans le processus délibératif nous aiderait à réduire la marginalisation systématique de ces groupes dans la société au sens large, autrement dit à accroître la justice des arrangements sociaux et politiques. La dynamique de la délibération à travers laquelle les perspectives des groupes marginalisés pourraient modifier la compréhension publique de la justice, de manière à favoriser le démantèlement des structures sociales inégales, devrait, je crois, se distinguer d'une certaine façon de la dynamique de la délibération que présupposent les modèles existants de la démocratie délibérative (que j'ai brièvement décrits plus haut). C'est ici qu'apparaissent mes inquiétudes quant aux limites du modèle délibératif.

Pour expliquer ma position, je vais d'abord esquisser un tableau de la dynamique qui caractériserait une politique délibérative incluant les voix des groupes marginalisés et servant la réforme des structures d'inégalité qui maintiennent leur identité. Au cours d'un tel processus, les membres des groupes marginalisés nous expliqueraient de quelle manière une pratique existante contribue à reproduire leur marginalisation et les empêche de participer au processus comme membres à part entière et égaux de la société. Par exemple, les femmes pourraient tenter de faire comprendre aux hommes comment le harcèlement sexuel entrave l'accomplissement de leur travail ou l'avancement dans leur carrière. Les individus ayant des handicaps pourraient tenter de nous expliquer tout le temps qu'il leur faut pour se déplacer dans un ville conçue sans tenir compte de leur existence, et comment la participation à certains champs de la vie sociale et du travail leur est tout simplement impossible, non pas à cause de la nature de leur handicap, mais parce qu'ils ne peuvent accéder physiquement aux lieux pertinents. Les autres participants à la discussion - les hommes, les personnes sans handicap - écouteraient leurs récits et apprendraient ainsi à envisager leurs propres pratiques sous un angle nouveau. $M$ ais il y a plus : afin que ces arguments aient un impact sur les structures sociales d'inégalité, leurs auditeurs devraient aussi se rendre compte du caractère opprimant et injuste de leurs propres pratiques. Finalement, ils devraient être prêts à changer ces pratiques, même si cela allait presque certainement à l'encontre de leurs intérêts tels qu'ils les ont compris jusqu'à présent. Iris Young décrit ainsi cette dynamique dans sa défense de la représentation de groupe:

La représentation de groupe dévoile le faux consensus que l'impérialisme culturel peut avoir produit. Elle révèle les préjugés de groupe dans les normes, les critères, les styles et les perspectives dont on a supposé l'universalité ou la plus haute valeur. En donnant voix à des besoins et à des expériences qui étaient réduits au silence ou dévalorisés, la représentation de groupe force les participants à la discussion à prendre une distance réflexive vis-à-vis de leurs présupposés et à penser au-delà de leurs propres intérêts. Lorsque des personnes sont confrontées à des intérêts, des besoins et des opinions qui sont dérivés de positions sociales et 


\section{$226 \cdot$ Philosophiques / Automne 2002}

d'expériences très différentes des leurs, elles peuvent parfois parvenir à comprendre les limites de leur propre expérience et de leur perspective dans la formulation d'une conclusion sur ce qui constitue la meill eure politique pour tous ${ }^{33}$.

$\mathrm{Si}$, toutefois, nous examinons de plus près la relation entre les postulats de la démocratie délibérative et les exigences d'un processus délibératif pouvant réformer les inégalités structurelles de groupe, nous découvrons que la différence sociale pose deux grands défis à la théorie délibérative. D'abord, I'attention portée aux perspectives distinctes sur des questions politiques qui suivent les lignes de clivage de la différence sociale soulève certains doutes quant aux critères du «raisonnable» de la théorie délibérative. Elle force aussi à s'interroger sur la façon dont les participants décident de ce qui compte comme raisons aux fins de la délibération politique. Certains critiques de la démocratie délibérative, partisans de la différence, considèrent que ses normes de raison publique sont elles-mêmes biaisées au détriment des modes de communication caractéristiques des groupes marginalisés. Q uoi que l'on pense de telles critiques, que je discuterai plus bas, il est clair que la reconnaissance des raisons des groupes marginalisés comme des raisons pour (ou acceptables pour) d'autres citoyens est quel que chose de très contingent : d'une part, parce que la propension de la délibération à réformer les inégalités structurelles de groupe dépend de la critique que les groupes marginalisés font des pratiques qui reproduisent leur désavantage; d'autre part, parce que ces critiques dépendent elles-mêmes d'interprétations contestées de la signification sociale de ces pratiques. De fait, il y a de bonnes raisons de croire que les groupes privilégiés auront systématiquement tendance à rejeter comme déraisonnables les interprétations des pratiques sociales que formulent les groupes marginalisés (même dans les cas où ce rejet n'est pas le produit d'une discrimination flagrante).

La seconde zone générale de tension entre les groupes marginalisés et la théorie délibérative découle naturellement de la première. Comme nous le verrons plus bas, les exigences d'un processus délibératif sensible à la différence sociale sont encore plus grandes que celles de la démocratie délibérative en général. Pourtant, la capacité de la démocratie délibérative à remplir sa promesse de produire des décisions politiques impartiales dépend de sa capacité à répondre à ces exigences. Si elle ne les satisfait pas, les théoriciens délibératifs devront admettre la tendance systématique des processus qu'ils recommandent à reproduire des inégalités systémiques injustes. Par conséquent, la théorie délibérative nous apparaîtra incomplète tant et aussi longtemps qu'elle négligera de formuler les conditions de réalisation de ses aspirations.

Le reste de cette étude s'intéresse à ces deux défis que pose la théorie de la différence sociale à la théorie délibérative.

33. Young, 1994b, p. 136. 


\section{Différence sociale et exigence de justice : deux problèmes pour la démocratie délibérative.}

Le critère du raisonnable et les normes de la délibération.

Certains critiques se sont intéressés aux contraintes discursives qu'imposent aux participants les théories de la démocratie délibérative. Ils considèrent que ces contraintes sont biaisées contre les groupes marginalisés, et ce de deux manières. Premièrement, disent-ils, certains modèles de démocratie délibérative exigent des participants qu'ils s'expriment en leur capacité de citoyens, formulant leurs arguments en termes d'intérêts généraux ou partagés, plutôt qu'à titre de porteurs d'intérêts et d'identités particuliers. Pourtant, ce n'est qu'en se concentrant sur la divergence d'intérêts entre groupes privilégiés et marginalisés que l'apport de ces derniers à la délibération peut contribuer au but de la justice envers ces groupes. Les participants à un processus délibératif sensible à la différence assument le rôle de porteurs d'intérêts distincts et identifiables, et non pas simplement celui (généralisé ou universalisé) de citoyens ${ }^{34}$. La diversité des perspectives qui sont invoquées dans la discussion des politiques publiques n'est pas seulement la diversité qui vient de ce que les participants maîtrisent différents courants de savoir et d'expérience. II s'agit plutôt d'une diversité qui reflète spécifiquement les structures sociales d'inégalité, les lignes de partage de la différence de groupe le long desquelles la position sociale, la richesse, les opportunités, l'influence politique, etc. sont inégalement distribuées. Dans la mesure où les normes discursives de la démocratie délibérative défavorisent les arguments fondés sur les intérêts partiaux, elles font obstacle à la justice ${ }^{35}$. A nne Phillips résume bien ce point : «Lorsqu'on demande aux groupes opprimés de mettre de côté leurs propres intérêts partiaux - afin de considérer les préoccupations communes à l'humanité entière, afin de penser au-delà de leurs propres besoins et intérêts - cette injonction peut les enfermer dans les structures mêmes qu'ils tentent de changer » 36 .

La plupart des théoriciens de la démocratie délibérative concéderaient aujourd'hui quel'obligation de généraliser les intérêts ne devrait pas s'appliquer à tous les niveaux d'argumentation, et que les arguments fondés sur des exigences de justice ont leur place légitime à l'intérieur de la délibération démocratique, qu'ils réfèrent ou non à une divergence d'intérêts entre citoyens. En d'autres termes, les citoyens partagent un intérêt dans la justice de leurs arrangements politiques, donc tout argument portant sur des considérations de justice appartient au forum public. De plus, comme l'affirment Gutmann et Thompson, « les individus et les groupes ne sont pas obligés de délibérer si d'autres refusent de le faire, et si leur participation à la délibération avait pour effet d'accroître leur désavantage. Les citoyens engagés dans des institutions coopératives n'ont pas d'obligations morales à faire leur part à moins

34. Voir Benhabib, 1992.

35. Voir notamment Young, 1990, p. 104-6.

36. Phillips, 1995, p. 147. 


\section{$228 \cdot$ Philosophiques / Automne 2002}

d'avoir l'assurance raisonnable que les autres ne prendront pas avantage d'eux $\gg 37$.

Une seconde critique lancée par les théoriciens de la différence contre la démocratie délibérative concerne la tendance de cette dernière à favoriser des formes d'expression qui ne sont ni caractéristiques des groupes marginalisés, ni bien adaptées à l'articulation de leurs perspectives : "Les normes de la délibération sont culturellement spécifiques et opèrent souvent comme des formes de pouvoir qui réduisent au silence ou dévalorisent la parole de certaines personnes »38. La spécificité culturelle des normes de délibération, selon Young, prend diverses formes. Contrairement aux suppositions de la théorie délibérative, la plupart des espaces de délibération réels sont le site de conflits, et non des lieux où les gens recherchent le consensus. De tels forums favorisent un discours «assuré et conflictuel » au détriment du discours «hésitant, exploratoire ou conciliant». Parce que ce dernier est le plus souvent caractéristique des groupes marginalisés que des privilégiés - et surtout, plus souvent le fait des femmes que des hommes - la délibération politique effective tend à favoriser des participants déjà privilégiés ${ }^{39}$.

La réplique des théoriciens délibératifs à ces critiques est qu'il existe amplement de talent et de compétence parmi les membres des groupes marginalisés pour produire des défenseurs efficaces de leurs positions. Ceci est particulièrement vrai, selon Gutmann et Thompson, dans les institutions représentatives où les participants à la délibération ont été choisis parmi un vaste bassin de citoyens: «les groupes désavantagés ont habituellement trouvé des représentants issus de leurs rangs qui pouvaient parler pour eux et formuler leurs intérêts et leurs idéaux, du moins aussi raisonnablement et efficacement que les représentants de groupes établis ${ }^{40}$.

$M$ ais Young va plus loin en affirmant que la théorie délibérative conçoit la communication démocratique de façon trop étroite, en selimitant à l'échange de raisons. Par conséquent, selon elle, la théorie délibérative forclot d'autres modes d'expression - incluant «la salutation, la rhétorique et le récit » - qui peuvent être plus propices à la compréhension à travers les frontières de la différence sociale que l'exposé d'arguments raisonnables. Young favorise, par conséquent, ce qu'elle appelle la « démocratie communicative» plutôt que la « démocratie délibérative», comme une théorie donnant une place centrale aux formes d'expression qui peuvent favoriser la réforme des inégalités structurelles de groupe. Par opposition aux normes d'offre de raisons de la démocratie délibérative, la démocratie communicative valide, selon elle, ces formes discursives ainsi que les aspects incarnés et affectifs de l'existence humaine qu'elles expriment. La démocratie communicative, précise-t-elle, s'engage à «privilégier éga-

37. Gutmann et Thompson, 1996, p. 72-73.

38. Young, 1996, p. 123.

39. Young, 1996, p. 123.

40. Gutmann et Thompson, 1996, p. 132-33. 
lement toute forme d'interaction communicative où les individus recherchent la compréhension. Si l'argumentation est un élément nécessaire de tels efforts de discussion et de persuasion mutuelle à propos de questions politiques, I'argumentation n'est pas le seul mode de communication politique» ${ }^{41}$.

Les théoriciens délibératifs répondent que le but de la politique démocratique doit être de satisfaire certains critères de légitimité dont la mesure est le caractère défendable des institutions publiques en termes de raisons que tous peuvent accepter, quelles que soient les formes d'expression nécessaires pour en arriver là. A lors que la rhétorique passionnée peut motiver les autres à écouter, et que les récits fortement évocateurs peuvent constituer un élément à l'appui des arguments du locuteur,

la tentative de Young de transformer le langage de l'État de droit dans un mode de communication plus partial, affectif et situé aurait pour conséquence d'introduire de l'arbitraire, car qui peut dire jusqu'où va le pouvoir d'une salutation? (...) la rhétorique émeut les individus et peut atteindre des résultats sans avoir à rendre compte des fondements à partir desquels elle amène les gens à s'engager dans certaines actions plutôt que dans d'autres ${ }^{42}$.

De plus, comme le suggère Victoria Kamsler, il n'y a aucune raison de supposer que la rhétorique puissante et la narration émouvante ne serviront pas les intérêts des groupes privilégiés autant sinon plus qu'elles ne servent les groupes marginalisés : «comme des blagues partagées, les histoires partagées peuvent avoir pour conséquence d'établir le fait d'une identité, d'une expé rience et d'un intérêt partagés [entre membres d'un groupe dominant], et elles ont ainsi joué un rôle important dans la rhétorique de [tels groupes] depuis Périclès» 43 .

Dans une veine similaire, Gutmann et Thompson prétendent que l'accent mis sur l'offre de raisons par la théorie délibérative ne bannit pas le discours passionné hors du forum politique. Ils admettent, en effet, que le discours passionné puisse jouer un rôle déterminant dans la délibération démocratique parce qu'on peut imaginer des circonstances dans lesquelles ce serait le seul moyen de mettre une question importante à I'ordre du jour délibératif. Ils prennent comme exemple le discours passionné de la sénatrice afro-américaine $\mathrm{C}$ arol M oseley-Braun au Sénat des États-Unis à propos d'une loi qui renouvelait le brevet protégeant l'emblème des $D$ aughters of the Confederacy, montrant le drapeau confédéré. M osel ey-Braun avait antérieurement persuadé le comité des affaires juridiques (judiciary committee) de ne pas renouveler ce brevet, mais les sénateurs J esse $\mathrm{H}$ elms et Strom Thurmond l'avaient inséré comme un amendement à un projet de loi de service national dont ils étaient confiants qu'il passerait. L'amendement passa lors d'un vote-test sans débats substantiels, malgréles protestations de M oseley-Braun et la présentation des

41. Young, 1996, p. 125.

42. Benhabib, 1996, p. 83.

43. Kamsler, 1996, p. 17. 


\section{$230 \cdot$ Philosophiques / Automne 2002}

arguments solides qu'elle avait exposés au comité des affaires juridiques. A la suite de ce vote, M osel ey-Braun se leva de son siège en menaçant I'obstruction et, «alliant raison et passion » ${ }^{44}$, elle lança un débat de trois heures sur les mérites de ce brevet. A cause de l'association étroite entre l'histoire de l'esclavage et le symbolisme du drapeau confédéré, cet amendement représentait, selon elle, «un outrage. C'est une insulte. II est absolument inacceptable à moi comme à des millions d'A méricains, noirs ou blancs, que nous donnions l'imprimatur du Sénat des États-U nis à un symbole de ce type d'idée». A la fin du débat provoqué par ce discours, I'amendement fut rejeté par une marge importante.

Gutmann et Thompson concluent de cet exemple que «les méthodes non délibératives peuvent s'avérer nécessaires à l'accomplissement des fins délibératives» : le Sénat n'avait pas considéré cette question de façon raisonnée jusqu'à ce que M oseley-Braun prenne passionnément la parole. Ils suggèrent de plus qu'un discours excessif peut communiquer d'importantes informations (dans ce cas, l'intensité des inquiétudes des A fro-américains au sujet du symbolisme du drapeau confédéré) plus efficacement que des arguments froids, calmes et raisonnés 45 .

Je trouve ces arguments persuasifs, et je suis ultimement d'accord avec les théoriciens délibératifs que l'idéal de la légitimité démocratique doit faire reposer les choix politiques sur des raisons acceptables pour tous les citoyens. N éanmoins, la place des perspectives des groupes marginalisés dans la délibération soulève des questions auxquelles les arguments de Benhabib et de Gutmann et Thompson ne répondent pas. Elle soulève en particulier des questions sur le critère du raisonnable au cœur de la théorie délibérative, sur la façon dont nous jugeons ce qui compte comme une raison dans la discussion politique. J'aimerais suggérer que le jugement du caractère raisonnable des arguments d'autrui est beaucoup plus contingent que ne le prétend la théorie délibérative, et que la contingence d'un tel jugement est fortement conditionnée par l'appartenance à des groupes structurés sel on les privilèges et les désavantages sociaux. Ce que les participants à la délibération pourraient accepter comme raisons peut dépendre, d'une manière importante, de qui ils sont et de qui leur présentent des raisons. Cela ébranle les prémisses de la démocratie délibérative, qui semble supposer implicitement que le caractère raisonnable ou déraisonnable des arguments d'autrui devrait être évident ${ }^{46}$.

Le statut des raisons comme raisons devient particulièrement problé matique dans les cas où l'objet du désaccord est la signification sociale des pratiques existantes, surtout lorsque ces pratiques renforcent (présumément) les structures injustes de privilèges sociaux actuels. Car c'est avant tout dans

44. Gutmann et Thompson, 1996, p. 136, citant «M s. M oseley-Braun's M ajestic M oment».

45. Voir Gutmann et Thompson, 1996, p. 135-36.

46. Sur le caractère problématique des critères du «raisonnable», voir M oore, 1996, p. 171-72. 
de telles circonstances que la signification sociale que les groupes marginalisés associent à une pratique diverge de la signification que lui donnent les groupes privilégiés. Dans ces circonstances, les raisons qui sous-tendent les critiques que formulent les groupes marginalisés de ces pratiques ne fonctionnent pas comme des raisons pour les membres des groupes privilégiés, car la signification sociale qu'ont ces pratiques pour les groupes marginalisés leur est (du moins initialement) inaccessible. Lorsque l'éradication des structures d'inégalité dépend du fait que ce soit la signification sociale que les groupes marginalisés donnent d'une pratique qui soit affirmée, et non pas celle qu'elle a pour les groupes privilégiés, la justice des résultats délibératifs dépend alors non seulement de la démonstration par les participants des vertus d'ouverture d'esprit et de respect mutuel (comme le souligne la théorie délibérative), mais également de la vertu de l'empathie ${ }^{47}$, et du fait de donner aux revendications des groupes marginalisés I'avantage particulier de cette empathie. II y a de bonnes raisons de croire que les groupes privilégiés seront prédisposés à percevoir comme déraisonnables les interprétations que font les groupes marginalisés des pratiques sociales lorsque ces interprétations divergent des leurs, particulièrement dans les cas où les interprétations que formulent les groupes privilégiés de ces pratiques ont pour effet de renforcer leur position d'avantage relatif (comme cela fut historiquement le cas, par exemple, pour les actes qui aujourd'hui portent le nom d' «harcèlement sexuel »).

Le caractère contingent de ce qui compte comme raison en délibération devient clair si l'on revient à l'exemple du débat au Sénat sur le brevet des Daughters of the Confederacy. L'appendice à cet essai résume ce débat tel qu'il a été rapporté par le Congressional Record. Une lecture attentive montre que tout reposait sur l'acceptation par une majorité de sénateurs de l'interprétation de M oseley-Braun de la signification sociale du drapeau confédéré (comme un emblème de l'esclavage) ou de celle, non-raciste, des sénateurs Blancs du Sud (comme remémoration des sacrifices à la nation). Alors que certains de ces derniers étaient sans doute malhonnêtes dans leur invocation d'une signification non-raciste du drapeau, il ne semble pas juste de les qualifier tous de racistes. Certains soutenaient sans doute une interprétation nonraciste du sens du drapeau et ne l'identifiaient pas à l'esclavage. Pourquoi la signification sociale que les Afro-américains donnent du drapeau devrait-elle compter comme une raison pour ces sénateurs, si le contenu de cette signification n'est pas celui qu'ils perçoivent ou soutiennent? La théorie délibérative ne semble pas, en l'état, pouvoir faire obstacle à ceux qui répondraient à M oseley-Braun, comme la Cour Plessy répondit aux arguments portant sur la honte de la ségrégation : «N ous considérons que le sophisme qui sous-tend l'argument du plaignant consiste dans la présupposition que la séparation forcée des deux races imprime à la race de couleur une marque d'infériorité. Si c'est le cas, ce n'est pas dû à quoi que ce soit que l'on retrouve dans la loi,

47. Pour une discussion connexe, voir Kymlicka, 1995a, p. 141, citant M inow, 1991. 


\section{2 - Philosophiques / Automne 2002}

mais seulement à la construction que la race de couleur choisit de lui appliquer ${ }^{48}$. Pourtant, des désaccords de ce type - sur la signification des pratiques sociales vécues comme opprimantes par les groupes marginalisés ont de fortes chances de faire partie des enjeux les plus importants du processus délibératif pour les groupes marginalisés. Si la démocratie délibérative n'offre aucune conception du critère que nous devrions utiliser pour juger de la réception par les groupes privilégiés des arguments qui portent sur les significations sociales, elle ne peut satisfaire le but de la justice envers les groupes marginalisés.

Cette difficulté n'a pas complètement échappé à $\mathrm{H}$ abermas et à R awls dans leur conception de la discussion politique, quoiqu'ils l'aient évoquée seulement en passant et qu'aucun d'eux n'ait proposé de solution. $\mathrm{H}$ abermas reconnaît qu'il y a des modes d'argumentation (incluant le mode esthétique) qui coexistent dans les discussions pratiques tout en ne visant pas l'accord rationnel. II concède que cette coexistence représente « une hypothèque sur l'exigence plus stricte de la discussion pratique (...), hypothèque qui découle de la situation socio-historique de la raison ${ }^{49}$. Pour moi, cela signifie que ce qui motive l'accord dans les discussions concrètes est un certain mixte d'appels à la raison, aux jugements esthétiques, etc., et que le mélange précis d'arguments pouvant générer un accord concret dépendra probablement des circonstances socio-historiques particulières qui entourent la discussion. De plus, $\mathrm{H}$ abermas reconnaît que le conflit social infléchit les discussions pratiques « dans la mesure où l'équilibre des rapports intersubjectifs de reconnaissance peut constamment varier en fonction des normes litigieuses» ${ }^{50}$. Encore une fois, ce point renforce mon argument selon lequel les perspectives des groupes marginalisés ne peuvent avoir d'influence sur les décisions politiques que si elles sont reconnues comme raisonnables par les autres participants. II existe pourtant de forts incitatifs pour que les groupes privilégiés refusent cette reconnaissance, surtout lorsque leurs intérêts bien établis sont en jeu (je reviens sur ce point dans la prochaine section). II y a donc de bonnes raisons de supposer que cette «hypothèque» des discussions pratiques, que représente leur propension à être affectées par des facteurs contingents d'identité sociale et de pouvoir social, a plus de chances d'être à la charge des participants marginalisés que des privilégiés dans une discussion.

L'idée que la raison sous-détermine le jugement politique est égal ement affirmée par John Rawls dans Libéralisme politique. Ceci n'a rien de surprenant; tous les défenseurs de la démocratie délibérative font une certaine place au « désaccord raisonnable» entre citoyens, même après qu'ils aient entrepris une discussion visant l'accord. Rawls nomme cette source de

48. Plessy v. Ferguson, 1896, p. 551; nos italiques.

49. H abermas, 1986, p. 127.

50. Ibid., 1986, p. 127. 
désaccord raisonnable les «difficultés de jugement». Parmi celles-ci, note Rawls, on retrouve le fait que:

Dans une certaine mesure (que nous ne pouvons préciser), la manière dont nous évaluons les preuves et pondérons les valeurs politiques et morales est modelée par notre expérience totale, l'ensemble de ce que nous avons vécu jusqu'à présent; or cela, nécessairement, sera toujours différent pour chacun. Ainsi dans une société moderne, comportant de nombreux emplois et positions, des divisions du travail différentes, de nombreux groupes sociaux et leur diversité ethnique, les expériences totales des citoyens sont suffisamment disparates pour que les jugements divergent (...) sur de nombreux cas relativement complexes, si ce n'est sur tous ${ }^{51}$.

En même temps, Rawls reconnaît aussi qu'il existe des «sources de désaccord déraisonnable», incluant «les préjugés, l'intérêt personnel et celui de groupe, l'aveuglement et l'entêtement $\gg^{52}$. M ais dans le contexte d'une discussion concrète dans laquelle il y a désaccord, comment les participants peuvent-ils distinguer entre un désaccord raisonnable fondé sur des expériences de groupe radicalement différentes et un désaccord déraisonnable fondé sur l'intérêt de groupe? A nouveau, le débat du Sénat concernant le drapeau confédéré nous présente une situation où ce qui est en jeu est précisément de savoir si l'interprétation que donne M oseley-Braun de ce symbole constitue une source de désaccord raisonnable ou déraisonnable; une représentation valide de I'expérience distincte des A fro-américains ou une tentative de marquer des points politiques en s'attaquant à un projet de loi inoffensif. Les ambiguïtés de cette affaire suggèrent que les « difficultés de jugement» peuvent retomber d'une façon disproportionnée sur les épaules des désavantagés.

II est clair, en tout cas, que les normes de délibération devant réguler les échanges entre groupes privilégiés et marginalisés diffèrent quelque peu des normes de la théorie délibérative telles que nous les avons discutées plus haut. En effet, à cause de la relation plus immédiate qu'entretiennent les participants à des intérêts identifiables, l'aspiration à l'impartial ité est beaucoup plus exigeante dans le second modèle de délibération que dans le premier (quoique ce dernier soit lui aussi très exigeant). M ettons-nous à la place d'un participant à la délibération dont les présupposés sont remis en cause par d'autres participants. Le premier modèle nous demande de prêter attention à la description que nous propose notre interlocuteur du bien commun et de l'évaluer dans un esprit d'impartialité, sans chercher à savoir si une telle conception servirait nos intérêts. Le second modèle nous demande d'aller plus loin, d'écouter avec sympathie sa prétention que nos pratiques les traitent injustement. II nous demande, en fait, d'écouter avec sympathie son assertion que la satisfaction de nos intérêts vient au prix d'une considération juste de leurs intérêts. Lorsque les arguments d'autrui sont solides, ce modèle exige que nous révisions

51. Rawls, 1995, p. 85-86; nous soulignons.

52. I bid., 1995, p. 86-87. 
notre évaluation de nos pratiques, que nous les jugions injustes, et que nous abandonnions les intérêts qu'elles servent au nom de la justice. A lors que dans le premier modèle nous pouvons «mettre entre parenthèses» la question de savoir comment nos intérêts pourraient être affectés par un changement de politique, nous ne pouvons éviter cette question dans le second modèle, puisque l'essentiel de l'assertion de notre interlocuteur est que les arrangements existants favorisent injustement nos intérêts au détriment des leurs. La rigueur de ces exigences morales conduit naturellement à se demander si la théorie de la communication démocratique qui en dépend ne serait pas désespérément idéaliste. Cela nous amène au second défi général qui se pose à la théorie délibérative du point de vue de la différence sociale.

Les conditions sociales et institutionnelles de la légitimité délibérative.

Bien que les participants en démocratie délibérative peuvent être situés également les uns par rapport aux autres, un processus délibératif qui vise l'atté nuation de l'inégalité structurelle doit faire des relations de pouvoir un enjeu central de l'ordre du jour délibératif. Ce n'est qu'en rendant visibles aux groupes privilégiés les conséquences injustes de leur pouvoir que les perspectives des groupes marginalisés peuvent avoir une chance de transformer les conceptions dominantes des exigences de justice. Pourtant, les modèles de démocratie délibérative restent généralement muets sur la relation entre les inégalités de pouvoir social et le processus de délibération. Cette omission remonte d'une certaine manière à l'influence de la théorie habermasienne sur les conceptions de la démocratie délibérative : la théorie de l'agir communicationnel de $\mathrm{H}$ abermas fait explicitement abstraction des relations de pouvoir afin de construire une «situation idéale de parole» libre des relations de pouvoir qui font obstacle à la «communication systématiquement non-déformée». Bien que rien dans la théorie délibérative n'empêche de donner priorité aux questions d'inégalité structurelle dans l'ordre du jour politique, reconnaissons que les théories de la démocratie délibérative ne donnent pas non plus de place centrale à ces questions dans leur conception de la légitimité politique. Plus important encore, elles ont tendance à négliger la question de savoir comment mettre sur pied des forums délibératifs dans lesquels citoyens privilégiés et marginalisés pourraient participer sur une base égale $\mathrm{e}^{53}$.

Gutmann et Thompson ont confronté ce problème de façon préliminaire et sans le résoudre. Comme ils le reconnaissent eux-mêmes :

II est peu probable que ceux qui profitent ... d'inégalités d'arrière-plan soutiennent des politiques qui viseraient à mitiger leurs effets. II est difficile de

53. Anne Phillips ressaisit bien ce problème : «les réflexions sur la démocratie délibérative ou communicative se réfèrent souvent de façon plutôt grandiose à un principe d'égal accès aux assemblées décisionnelles ou à l'égalité substantielle de ressources et de pouvoir, mais elles ne donnent que peu d'attention soutenue à la question de savoir comment ces conditions pourraient être jamais assurées » (Phillips, 1995, p. 154). 
délibérer sans passion sur des lois qui pourraient mettre un terme à nos propres chances de délibérer comme législateur. Les résultats de la représentation délibérative sont faussés, mais pas pour autant déterminés, par les mêmes inégalités que le processus délibératif de représentation est supposé corriger ${ }^{54}$.

Leur défense de la démocratie délibérative envers cette critique est que «la délibération doit faire partie de toute stratégie visant à sortir du cycle d'injustice créé par les inégalités d'arrière-plan ». Ils ajoutent que les institutions législatives actuelles ont des caractéristiques qui donnent de l'importance à des facteurs autres que le statut social - commel'habileté politique, la collégialité, et l'esprit même de réciprocité - dans la détermination des chances qu'a un individu donné d'être entendu de façon équitable par ses collègues ${ }^{55}$. Ces remarques ont un certain mérite, mais on pourrait se demander si la théorie de la démocratie délibérative peut être complète sans une élaboration supplémentaire des circonstances - autant sociologiques qu'institutionnelles dans lesquelles les processus délibératifs peuvent éviter les préjugés structurels contre des groupes déjà désavantagés.

Tout bien considéré, le résultat du processus délibératif qui répond spécifiquement à la différence de groupe est impartial en un sens différent de l'impartialité des résultats dans les modèles existants de la démocratie délibérative. Ce résultat reste impartial au sens où il ne favorise pas certains groupes au détriment des autres, mais il va plus loin en ce qu'il doit être aussi transformateur ; il doit corriger les partis-pris passés des arrangements sociaux selon des modalités que les autres modèles de démocratie délibérative considèrent rarement.

La critique de Cass Sunstein de ce qu'il appelle la « neutralité de statu quo » constitue à cet égard une exception importante dans la littérature sur la démocratie délibérative. Comme le signale Sunstein, une bonne partie du discours libéral et juridique contemporain « définit la neutralité en prenant le statu quo, ou ce que divers groupes ou personnes ont maintenant, comme un donné et comme base de la décision. (...) S'éloigner du statu quo témoigne d'un esprit partisan; respecter le statu quo témoigne de neutralité ${ }^{56}$. M ais nous n'avons pas à accepter cette présomption en faveur des pratiques et des distributions existantes, même dans les cas où il y a désaccord sur les pratiques alternatives qui seraient les plus désirables. A u contraire, lorsque le statu quo fixe des inégalités injustifiables entre groupes, le maintenir constitue une injustice. Un engagement en faveur de la démocratie délibérative, qui requiert la défense des pratiques politiques en des termes justifiables pour tous les citoyens, implique l'abandon de la neutralité de statu quo ${ }^{57}$.

54. Gutmann et Thompson, 1996, p. 134.

55. I bid., 1996, p. 134.

56. Sunstein, 1993, p. 3.

57. Sunstein, 1993, p. 6, 353. 


\section{6 - Philosophiques / Automne 2002}

Pour un certain nombre de raisons, la conception de la délibération qui émerge d'une réflexion centrée sur la contribution qu'offre à une conception collective de la justice une discussion impliquant les groupes marginalisés apparaît plus radicale que ce que semblent admettre les versions plus courantes de la démocratie délibérative. Une politique délibérative de la différence n'a de sens que comme une politique transformatrice, qui considère non seulement « une distribution plus équitable des ressources et du pouvoir entre des groupes dont nous estimons qu'ils demeureront hostiles», mais également l'émergence d' « une conception plus complète, qui confirme la valeur de chaque groupe ${ }^{58}$. M ais en nous offrant une conception plus radicale de la démocratie que la plupart des autres modèles délibératifs, elle semble également courir le risque d'être encore plus naïvement utopique que ces derniers, lesquels ont été eux-mêmes fréquemment critiqués pour leur manque de réalisme concernant la psychologie et les motivations humaines, parce qu'ils affirment « des présupposés héroïques au sujet des participants» 59 . Si les participants à la démocratie délibérative tels qu'ils sont normalement décrits sont « héroïques», il semblerait alors que les participants à une politique délibérative de la différence doivent être divins ${ }^{60}$.

En fait, je ne crois pas que l'idéal de la politique délibérative de la différence soit naïvement utopique en toutes circonstances; mais nous devons être plus spécifique quant aux circonstances dans lesquelles on pourrait concevoir que les groupes privilégiés abandonnent certains de leurs privilèges en réponse à des revendications de justice des groupes marginalisés. $M$ ais alors, qu'est-ce qui pourrait susciter chez les groupes privilégiés une disposition à écouter impartialement les revendications des groupes marginalisés de la façon que j'ai décrite?

II me semble qu'il y a deux sources principales de motivation pour les puissants à écouter avec empathie les voix des plus faibles : (a) le désir d'être juste, ou du moins de pouvoir justifier sa position aux autres (autant au sens de nos croyances que dans celui de notre statut social) ${ }^{61}$; et (b) le besoin d'endiguer le conflit afin d'en éviter les coûts. Ces deux sources peuvent motiver les puissants à considérer et à tenter de comprendre une situation du point de vue de l'autre, même moins puissant. D e fait, si nous examinons les moments historiques les plus essentiels de l'inclusion des groupes opprimés au sein de la politique démocratique - les amendements concernant les droits civils, l'amendement portant sur le droit de vote des femmes, la loi sur les droits civils, la démocratie sud-africaine, l'inclusion des Premières nations dans les négociations constitutionnelles canadiennes - tous ont en commun

58. Phillips, 1993, p. 160.

59. Johnson, 1995, p. 25-26.

60. Cf. La description de R ousseau de l'auto-gouvernement démocratique: « S'il y avait un peuple de dieux, il se gouvernerait démocratiquement. Un gouvernement si parfait ne convient pas à des hommes» (Rousseau, 1966, p. 108).

61. Voir Scanlon, 1982. 
le fait qu'une ouverture nouvelle aux groupes opprimés s'accompagne autant d'une incapacité de pouvoir justifier les pratiques courantes que d'une mobilisation sociale des opprimés menaçant de coûts potentiellement élevés les groupes et/ou classes dominants s'ils échouaient à résoudre les conflits.

Cela suggère toutefois que les sources de motivation que sont le bien commun de la justice et l'intérêt sont en fait liés beaucoup plus étroitement que ne l'admettent les modèles dominants de la démocratie délibérative. C'est le fait de voir leurs intérêts menacés qui incline parfois les groupes privilégiés à accepter une redéfinition des exigences de justice. Bien sûr, le même rapport entre intérêt et justice existe aussi pour les groupes marginalisés, bien qu'il apparaisse sous une autre forme : comme plusieurs opposants à la reconnaissance de groupe l'ont noté, accepter les demandes de justice fondées sur l'existence de groupes crée une motivation pour ceux-ci à formuler des revendications malhonnêtes et à utiliser le statut de « victime» comme un instrument de pouvoir politique. Voilà une dynamique dont je ne souhaite pas nier l'existence, même si jen'en tire pas la même conclusion que les adversaires de la « politique de la différence».

L'analyse des tensions entre les exigences de représentation formulées par les groupes marginalisés et l'idéal de la démocratie délibérative nous permet de dégager quelques lacunes importantes de ce dernier. Plus particulièrement, il semble que les modèles de la démocratie délibérative resteront des outils imparfaits pour l'approfondissement de notre compréhension de l'égalitarisme démocratique et pour sa promotion tant que leur caractérisation des normes de délibération et du rapport entre participants demeurera dans le domaine de l'idéal théorique. Ces modèles ne pourront non plus atteindre leurs buts tant qu'ils supposeront l'existence d'une disjonction nette entre la politique des intérêts et la politique délibérative. $\mathrm{N}$ otre analyse suggère que c'est plutôt sous la forme d'un continuum entre la solidarité parfaite et la lutte débridée des intérêts que nous devrions nous représenter la politique.

Si nous choisissons de penser ainsi la politique et les possibilités d'une délibération égalitaire, je crois que nous allons nous rendre compte que la capacité des processus politiques de s'approcher de l'idéal d'impartialité dépend crucialement (a) des circonstances sociales, particulièrement du degré de mobilisation des groupes marginalisés et (b) de la structure des institutions politiques. Ces deux facteurs peuvent exercer une pression sur les groupes privilégiés qui pourrait les conduire à coopérer sur un mode délibératif avec les groupes moins privilégiés. La mobilisation politique des groupes marginalisés crée de la pression de la manière décrite plus haut. Les institutions politiques peuvent elles-mêmes fournir des incitatifs à la délibération par des règles de décision qui se rapprochent du consensus ou de l'unanimité comme condition des décisions politiques légitimes ou contraignantes (comme dans le système des majorités concurrentes de John C. Calhoun, ou les règles de majorité qualifiée). Elles le peuvent aussi par les normes mêmes de la discussion publique. Comme Jon Elster l'a récemment proposé, la publicité de 


\section{8 - Philosophiques / Automne 2002}

I'argumentation politique peut, en elle-même, avoir pour effet de modérer les jeux d'intérêts dans la prise de décision politique, même lorsque ce sont ces intérêts qui motivent les participants, une dynamique qu'il décrit comme la «force civilisatrice de l'hypocrisie» 62 .

Récemment, certains théoriciens de la démocratie délibérative ont pris le parti de quitter le champ de la théorie idéale pour esquisser une théorie de la démocratie qui soit réceptive aux imperfections des sociétés dans lesquelles on espère pouvoir améliorer la délibération politique ${ }^{63}$. A lors que la théorie idéale occupe une place importante dans notre compréhension de la justice et nos aspirations à l'impartialité, l'accent mis sur les exigences d'une politique délibérative de la différence met en évidence certaines des limites inhérentes à la théorie idéale. Elle nous conduit à nous intéresser aux questions de design institutionnel et à rechercher des généralisations empiriques sur les relations historiques entre les groupes privilégiés et marginalisés ${ }^{64}$. Si les efforts récents des théoriciens de la démocratie d'aller au-delà de la théorie idéale sont louables, beaucoup de travail reste à faire.

\section{Conclusion}

Dans cette étude, je suis partie d'une position sur la citoyenneté selon laquelle la citoyenneté pleine et égale des membres des groupes marginalisés dépend de leur participation aux processus de décision politique; ces processus devant eux-mêmes se montrer ouverts aux raisons invoquées par les groupes marginalisés. Les institutions elles-mêmes peuvent être réformées jusqu'à un certain point, afin d'encourager une délibération plus inclusive entre citoyens. $M$ ais I'inclusion au sein des institutions décisionnelles ne garantit pas en soi que les résultats politiques refléteront les besoins et les préoccupations des citoyens provenant des groupes marginalisés. Les arguments que j'ai formulés ici soutiennent la notion familière que la justice et la légitimité des sociétés démocratiques dépendent autant des qualités des citoyens que du design des institutions.

Plus important encore, la capacité qu'ont les processus de délibération démocratique de réformer l'injustice structurelle envers les groupes marginalisés dépend de la volonté des citoyens d'écouter les arguments d'autrui dans un esprit d'impartialité. Comme je l'ai montré, cette capacité est encore plus exigeante que ne le supposent les démocrates délibératifs. Pour des citoyens relativement privilégiés qui entrent en discussion avec des citoyens marginalisés, cela suppose la volonté d'interroger leurs propres jugements concernant le caractère déraisonnable des arguments d'autrui, surtout lorsque la reconnaissance de la validité de ces arguments pourrait mettre en péril leurs intérêts matériels ou culturels. On pourrait même aller jusqu'à dire qu'une discussion politique

62. Elster, 1995.

63. Voir notamment Cohen et Rogers, 1995; Gutmann et Thompson, 1996; H abermas, $1996 a$.

64. Pour une description instructive de la relation entre théorie idéale et non-idéale, voir Carens, 1996. 
impartiale requiert parfois, dans son esprit, une sorte de «principe de différence appliqué aux raisons» 65 . J 'entends par là la volonté de considérer les raisons des groupes marginalisés comme des raisons qui soutiennent une décision collective même lorsqu'elles ne nous sont pas immédiatement disponibles à partir de notre propre expérience sociale ou culturelle. Comme l'atteste le débat au Sénat à propos du drapeau confédéré, le discours de Carol M oseley-Braun a motivé bien des sénateurs Blancs à adopter précisément ce comportement.

$\mathrm{M}$ a conception de la délibération démocratique avance également une relation empirique entre le niveau de mobilisation sociale et politique d'un groupe et la réceptivité de la discussion démocratique à l'expérience distincte de ce groupe. Si une telle relation existe, cela comporte des implications supplémentaires pour notre compréhension des vertus du citoyen. L'apathie politique et la passivité réduisent la légitimité du processus démocratique pour tous les citoyens, mais la réalisation de la justice démocratique envers les citoyens marginalisés apparaît particulièrement dépendante de leur implication active non seulement dans les principales institutions de décision politique, mais aussi dans les mouvements sociaux ${ }^{66}$. Un engagement envers la réforme des inégalités structurelles de groupe entraîne donc avec lui un engagement en faveur d'une participation démocratique accrue des citoyens défavorisés.

\section{Appendice}

La sénatrice Carol M oseley-Braun et le débat sur le drapeau confédéré

Le 12 mai 1993, le comité des affaires juridiques (judiciary committee) du Sénat américain, dont la sénatrice $C$ arol $M$ osel ey-Braun était membre, évalua la demande d'une importante organisation sociale et caritative du Sud des États-Unis, la United D aughters of the Confederacy (UDC) pour le renouvellement d'un brevet spécial concernant son insigne. Celui-ci incluait une image du drapeau confédéré. L'appartenance à l'organisation est basée sur les liens ancestraux avec ceux qui ont combattu du côté de la Confédération lors de la Guerre civile américaine. M oseley-Braun avait entrepris une recherche considérable sur cet insigne et les liens entre la cause de la Confédération et l'histoire de l'esclavage aux États-Unis. Elle s'opposa au renouvellement du brevet pendant les débats du comité et présenta le résultat de ses recherches, à savoir que l'insigne était amplement protégé par une autre loi portant sur les brevets, et que l'attribution par le Sénat d'un brevet spécial constituait une mesure rare et

65. En référence à la seconde partie du second principe de justice distributive de John Rawls, qui stipule que « les différences socio-économiques liées à des fonctions et à des positions doivent être ajustées (...) au plus grand bénéfice des membres les plus désavantagés de la société» (Rawls, 1995, p. 31).

66. Le type d'activisme politique que j'ai à l'esprit inclut l'organisation des citoyens dans ce que $\mathrm{N}$ ancy Fraser appelle «les contrepublics subalternes», soit des sous-cultures sociales à l'intérieur desquelles les groupes marginalisés développent une compréhension d'eux-mêmes et une force organisationnelle permettant l'entrée dans les institutions politiques définies par la constitution (Fraser, 1997a, p. 80-85; voir aussi M ansbridge, 1996). 
extraordinaire. Le comité vota à 13 contre 2 contre le renouvellement du brevet. Par la suite, le sénateur J esse $\mathrm{H}$ elms de la Caroline du N ord, en catimini, ajouta le brevet à un projet de loi sur le service national. Le 22 juillet, le vote sur l'amendement surgit sans préavis. M oseley-Braun, qui n'avait pas anticipé l'amendement, décida sur l'impulsion du moment qu'elle devait s'y opposer au Sénat, pour les mêmes raisons qu'elle s'y était opposée en comité.

Le discours d'ouverture de M oseley-Braun culmina en une motion d'ajourner l'amendement, c'est-à-dire de le supprimer du projet de loi. Elle commença en soulignant que l'emblème de I'UDC ne requérait pas la protection d'un brevet approuvé par le Sénat, parce qu'il était déjà amplement couvert par la législation générale portant sur les brevets. Le travail de cette organisation ne dépendait pas non plus, d'aucune façon, du fait de disposer d'un tel brevet. Elle souligna que protéger un emblème par un brevet «n'est pas seulement une question de simple reconnaissance. C'est un honneur rare conféré à une organisation ». Elle insista sur le fait que moins de dix organisations l'avaient reçu au cours du dernier siècle.

Les U nited D aughters of the Confederacy ont le droit d'honorer leurs ancêtres et de choisir le drapeau confédéré comme leur symbole si elles le souhaitent. Cependant, ceux parmi nous dont les ancêtres ont combattu dans un camp différent lors de la Guerre civile, ou qui étaient, disons le franchement, détenus comme de la propriété humaine sous le drapeau confédéré, ont aussi le devoir d'honorer leurs ancêtres en demandant si une telle reconnaissance par le Sénat des États-Unis est appropriée ${ }^{67}$.

Les membre de l'organisation, selon elle,

ont le droit d'utiliser l'emblème de leur choix, il ont le droit de s'organiser de la manière qu'ils le désirent, ils ont le droit d'entreprendre ce qu'ils veulent. $M$ ais, en même temps, il est inapproprié pour ce Sénat, pour ce Congrès des États-Unis, de conférer un imprimatur spécial, extraordinaire, si vous voulez, à un symbole qui est aussi inapproprié pour nous tous A méricains que l'est ce symbole ${ }^{68}$.

$M$ oseley-Braun souligna le fait que c'était à cause du symbolisme raciste du drapeau que le Sénat devait refuser de l'endosser.

Le fait est que les emblèmes de la Confédération ont un sens pour les A méricains même cent ans après la fin de la Guerre civile. Tout le monde sait ce que la Confédération représente. Tout le monde sait ce que ces emblèmes représentent (...). Lorsqu'en 1963 un ancien Gouverneur se leva et fit flotter le drapeau de bataille de la Confédération au mat du Capitole de l'État de l'A labama afin de protester contre le soutien par le G ouvernement fédéral des droits civils et contre la visite du solliciteur général de l'époque, tout le monde savait ce que cela voulait dire. M aintenant, en 1993, lorsque nous voyons ressortir les symboles de la Confédération, tout le monde sait ce que cela veut dire ${ }^{69}$.

67. Congressional Record, 22 juillet 1993, s9253.

68. I bid., 59253.

69. Ibid., s9253-54. 
Elle poursuivit en insistant sur l'importance de se concentrer sur les symboles qui unissent les A méricains plutôt que sur ceux qui les divisent :

La bannière étoilée est pour toujours notre drapeau, que nous soyons du $\mathrm{N}$ ord ou du Sud, que nous soyons ou non des Afro-américains - voilà notre drapeau. Et donner un brevet, ce que même notre drapeau ne possède pas, à un symbole de la Confédération, me semble simplement créer, au sein de notre société, le genre de divisions qui sont contre-productives ${ }^{70}$.

M oseley-Braun inclut au procès-verbal du Sénat une lettre du U.S. Patent $\mathrm{O}$ ffice confirmant ses arguments sur la rareté de tels brevets et la protection des insignes sous d'autres dispositions légales.

À la suite de l'argumentation de M oseley-Braun et la présentation de ses preuves, il y eut un débat entre les sénateurs Strom Thurmond de la Caroline du Sud et $\mathrm{H}$ oward M etzenbaum de l'O hio sur la protection offerte par le brevet à l'insigne de l'organisation : répondait-il ou non à un besoin quelconque? H elms intervint en prétendant que la motion d'ajourner l'amendement constituait une «action punitive» et un «abus» pour lequel «ces bonnes dames» avaient été «distinguées ${ }^{71}$. La sénatrice Patti M urray de Washington se leva par solidarité pour M oseley-Braun, invoquant leur position commune de femmes au Sénat et notant que M oseley-Braun était la seule Afro-américaine de cette institution : «N ous n'allons pas laisser la déférence à certaines idées de tradition cacher le racisme ou toute autre forme de discrimination ou d'intolérance» 72 .

À ce moment du débat, on tint un vote sur la motion de M oseley-Braun d'ajourner l'amendement; celle-ci fut rejetée à 52 contre 48.

A près le vote, l'enceinte du Sénat se remplit de murmures, alors que M oseley-Braun se levait de nouveau pour prendre la parole, cette fois-ci sur un ton moins modéré. M oseley-Braun déclara, à travers le bruit, «Je dois vous dire que ce vote porte sur la race (...). II porte sur des symboles raciaux, sur le passé racial, et sur l'épisode le plus douloureux de l'histoire américaine». Une fois le Sénat rappelé à l'ordre, M oseley-Braun continua à parler du drapeau confédéré, devenant toujours plus passionnée. Elle devint tellement agitée qu'elle sentit le besoin de s'excuser :

Je suis désolée, $M$ adame la Présidente. Je vais baisser le ton. Je deviens excédée parce que, pour le dire très franchement, voilà ce qui est vraiment en cause. La question est de savoir si oui ou non des A méricains comme moi, qui croient dans la promesse de ce pays, qui ont pour lui un fort attachement, qui sont des patriotes de ce pays, devront avoir à souffrir l'indignité de se voir rappeler constamment, qu'à un certain moment dans l'histoire de ce pays, nous étions de la propriété humaine. N ous étions des choses. N ous pouvions être échangés, achetés, et vendus. Suggérer maintenant, comme le ferait une histoire révision-

70. I bid., s9254.

71. I bid., s9256.

72. I bid., s9256. 
niste, que ce drapeau n'a rien à voir avec l'esclavage, voilà qui heurte de plein fouet l'histoire.

Elle souligna qu'elle avait tenté, dans son discours précédent, d'être «froide» plutôt qu' «incendiaire», et qu'elle avait consciemment choisi de ne pas présenter une partie de ses recherches sur le drapeau confédéré et ses liens symboliques avec l'esclavage. $M$ ais elle ne voyait plus maintenant aucune raison pour une telle modération, et elle lut des extraits d'un discours de 1861 du Vice-président de la Confédération intitulé «L'esclavage, pierre angulaire de la Confédération», dans lequel il affirmait : «N otre nouveau gouvernement est fondé sur (...) la grande vérité que le nègre n'est pas l'égal de I'homme blanc, que l'esclavage, la subordination à la race supérieure, est sa condition naturelle et morale. » M oseley-Braun poursuivit : « C'est un outrage absolu que cette institution pourrait adopter comme amendement à cette législation un symbole de ce point de vue». Elle insista qu'elle avait tenté, en sept mois de présence, d'adopter une attitude conciliante envers ses collègues, « $M$ ais je vous dis, $M$ adame la Présidente, que sur cette question il ne peut y avoir de consensus. C'est un outrage. C'est une insulte. II est absolument inacceptable à moi comme à des millions d'A méricains, noirs ou blancs, que nous donnions l'imprimatur du Sénat des États-Unis à un symbole de ce type d'idée». Elle blâma les membres de son propre parti pour avoir voté en faveur de l'amendement.

M oseley-Braun pressa ses collègues des deux partis de prêter à l'amendement toute l'attention qu'il méritait et de reconsidérer leur jugement qu'il n'y avait pas là « de quoi faire un plat». Elle les pressa de prendre conscience du fait:

qu'il y a là, en effet, de quoi faire un très grand plat - que l'imprematur qui est ici recherché aujourd'hui lance un message au reste du pays que cette étrange institution n'a pas été mise au rancart une fois pour toute; qu'en effet, comme Dracula, elle revient nous hanter encore et encore et encore; et que malgré les progrès que nous avons accomplis, le fait est que ceux-là existent qui voudraient nous faire glisser à nouveau dans l'obscurité de la division, dans la fosse aux serpents de la haine raciale (...) et du soutien de symboles de la lutte pour garder les Afro-américains (...) en esclavage ${ }^{73}$.

Certains collègues $D$ émocrates de $M$ oseley-Braun commencèrent à se joindre au débat et à soutenir ses arguments. Dianne Feinstein, de la Californie, appela notamment ses collègues du Sud à écouter les commentaires de M oseleyBraun «à propos de l'impact du drapeau confédéré (...) sur une portion importante de l'électorat américain, ceux qui sont Afro-américains; ceux qui portent avec eux I'héritage d'une nation qui, à une certaine époque et dans une grande mesure toujours aujourd'hui, a un certain racisme, un certain préjugé dans une bonne part de nos actes (sic) ${ }^{74}$. Bill Bradley s'exprima aussi en faveur de

73. Ibid., s9258.

74. Ibid., s9258. 
M oseley-Braun et pressa ses collègues de reconsider leur vote. II rappela également aux R épublicains qu'ils appartenaient au parti de Lincoln, et il demanda à tous ses collègues de ne pas écarter le discours de la sénatrice à cause de son émotion : "C'est émotif. C'est émotif comme tout ce qui touche au plus profond de notre humanité et comme tout ce qui espère que le meilleur de nousmêmes, en tant qu'A méricains, l'emportera chaque jour » 75 .

M oseley-Braun intervint à nouveau dans le débat, rappelant au Sénat que l'amendement avait été défait en comité par un vote de 13 à 2 et elle dit à quel point elle avait été surprise de le voir attaché au présent projet de loi. La stratégie des parrains de l'amendement avait été de supposer qu'il passerait inaperçu. Cette conviction, affirma-t-elle, était le reflet d'un échec à «comprendre les implications de ce qu'il signifie, non pas seulement pour moi personnellement, mais pour chaque descendant de cette étrange institution. » Le sénateur Exon, du N ebraska, intervint à cet instant pour soutenir que l'enjeu n'était pas simplement une question de racisme:

Je sais très bien que la plupart de ceux qui ont voté contre la motion d'ajournement ne l'ont pas fait (...) en leur cœur et conscience pour des raisons raciales. J e soupçonne plutôt que c'était à cause d'une vieille attitude du Sud - le Sud reviendra (...). Je soupçonne en toute sincérité que la vaste majorité de ceux qui n'ont pas voté en faveur de la motion d'ajournement n'avait aucune idée ou compréhension du contexte tout à fait légitime et des préoccupations qui ont été exprimées par la sénatrice de l'Illinois ${ }^{76}$.

M ais cela, poursuivit-il, ne devrait pas empêcher le Sénat de reconnaître $q^{\prime}{ }^{\prime} \ll i l$ avait commis une erreur .

Les sénateurs Démocrates $\mathrm{M}$ oynihan, de $\mathrm{N}$ ew York, et Biden, du $\mathrm{N}$ ew J ersey, intervinrent ensuite pour souligner l'importance capitale du discours de M oseley-Braun, insistant que celui-ci les avait conduits, ainsi que d'autres, à percevoir une dimension de l'expérience afro-américaine dont ils n'étaient pas conscients auparavant. M oynihan affirma que ce discours était le plus émouvant qu'il ait entendu en 17 ans au Sénat, et qualifia d' « épiphanie» la vision qu'elle avait de la signification du drapeau confédéré ${ }^{77}$. Biden souligna sa collégialité, son absence de dogmatisme et sa capacité à agir comme une force de conciliation au Sénat. II poursuivit en disant que son discours démontrait l'importance de la présence des minorités au Sénat :

J e pense que nous avons vu ici aujourd'hui dans I'enceinte du Sénat une des raisons pour lesquelles je dis avec d'autres et depuis si longtemps que cette institution a besoin de diversité, (...) d'être capable, que ce soit à titre d'A méricain d'origine autochtone, d'Afro-américain noir (sic), d'Irlandais-américain, ou encore d'A méricain d'origine asiatique, d'apporter ce dont ce pays a le plus 


\section{4 - Philosophiques / Automne 2002}

besoin en ce moment, et c'est la civilité et une certaine compréhension du point de vue de l'autre ${ }^{78}$.

La sénatrice de l'Illinois a montré quelque chose aujourd'hui qui a cruellement manqué dans cette institution : qu'une seule voix parlant pour des millions et des millions de voix dans ce pays qui se sentent exclues, et qui le sont dans plusieurs cas, qui sentent que cette institution ne comprend pas leurs problèmes, et dans plusieurs cas c'est vrai ; qui savent que la plupart d'entre nous ne viennent pas d'un contexte ou de circonstances qui nous permettent non seulement de comprendre, mais de sentir et de goûter les problèmes d'un segment entier de ce pays. Ceux-ci ont finalement une voix qui a deux fonctions, non seulement de représenter leur point de vue, mais de nous sensibiliser à ce point de vue, parce que, on peut l'espérer, nous partageons tous ici la vision réconciliatrice de la sénatrice de l'Illinois ${ }^{79}$.

Le sénateur Ben $\mathrm{N}$ ighthorse-Campbell, le seul sénateur amérindien, intervint également afin d'exprimer sa solidarité envers M oseley-Braun, à titre de membre d'un groupe minoritaire qui continue à être l'objet de discrimination et de racisme.

A ce moment du débat, des sénateurs Républicains commencèrent à se joindre de nouveau à la discussion. Le sénateur Simpson, du Wyoming, qui avait voté contre la motion de M oseley-Braun, nota que le brevet avait été unanimement renouvelé à quatre reprises au cours du siècle. II demanda à $M$ oseley-Braun si celle-ci pensait que le Sénat, en renouvelant le brevet tant de fois, $n$ 'avait pas commis « une forme quelconque de décision raciste». M oseley-Braun répondit en niant tout mobile raciste de la part des Sénats présent ou passés, affirmant que : «C ela s'est simplement produit. Cela s'est simplement produit parce qu'il n'y avait personne pour attirer l'attention sur ce qui était clairement et évidemment une erreur $\gg 80$.

La prochaine intervention fut un discours émouvant et un point tournant du débat. Le sénateur $\mathrm{H}$ owell $\mathrm{H}$ eflin de l'A labama commença en invoquant son amour du Sud et ses propres racines familiales dans la Confédération, de même que ses liens familiaux avec les D aughters of the Confederacy. II souligna qu'il ne croyait pas que l'organisation ait des intentions racistes. II rapporta une remarque qu'il avait faite à son directeur législatif, dont l'arrière-arrièregrand-père avait été un esclave : «si je vote avec le sénateur M oseley-Braun, ma mère, ma grand-mère, et d'autres ancêtres se retourneront dans leurs tombes». À quoi son directeur lui répondit : «Bien, de la même façon, mes ancêtres se retourneront dans leurs tombes [si vous ne le faites pas]». « M ais toute l'affaire se réduit à ce que la sénatrice $M$ osely-Braun soutient - à savoir que c'est une question de symbolisme. $\mathrm{N}$ ous devons laisser le racisme derrière nous, et nous devons avancer ». II dit qu'il soutenait, par conséquent, une reconsidéra-

78. Ibid., s9260.

79. I bid., s9261.

80. I bid., s9262. 
tion de la motion d'ajourner l'amendement: «J e sens qu'aujourd'hui ceci est un pas symbolique. Si nous décidons d'apposer le sceau d'approbation du Sénat des États-Unis et du Congrès sur un symbole qui offense une large section des Américains, je pense que nous n'avancerons pas dans la bonne direction, et que c'est là une approche erronée des idéaux que ce pays doit défendre » ${ }^{81}$.

A près le discours de $\mathrm{H}$ eflin, d'autres sénateurs qui avaient voté contre $M$ oseley-Braun commencèrent à changer de bord. Le sénateur $\mathrm{M}$ cConnell du Kentucky affirma : "Je n'avais jamais pensé en votant que ce serait interprété comme, en quelque sorte, une approbation du racisme ou de l'esclavage. Je l'ai fait plutôt par respect pour mes ancêtres et pour leurs racines qui s'enfonçaient profondément dans le Sud ${ }^{82}$. Le sénateur Riegle du $M$ ichigan expliqua son revirement par la force des discours de M oseley-Braun et de $\mathrm{H}$ eflin. II invoqua le service militaire des Afro-américains et suggéra que lorsque des citoyens ont mis leurs vies en danger pour la nation, ils ne devraient pas être repayés en symboles qu'ils considèrent dégradants.

Plusieurs sénateurs Démocrates entrèrent à nouveau dans la discussion pour soutenir M oseley-Braun. Le sénateur Kerry du M assachusetts affirma :

Ce qui me frappe, alors que je me tiens devant vous, c'est qu'il y a un vrai malaise quand je pense au fait que nous sommes 96 hommes et femmes blancs en train de débattre si oui ou non nous devrions être sensibles à l'expression d'un Afro-américain et d'un A méricain amérindien. Si cela ne nous dit pas quel est le problème, alors rien ne le fera ${ }^{83}$.

La sénatrice Barbara Boxer de la Californie parla des remarques du sénateur $\mathrm{H}$ eflin en termes élogieux.

Car ce qu'il a fait aujourd'hui, et la manière dont il l'a fait, s'adresse à ce qu'il y a de meilleur en chaque Américain. S'il y a jamais eu une preuve de l'importance de la diversité dans cette institution, nous avons reçu cette preuve aujourd'hui. Je crois vraiment que si la sénatrice de l'Illinois n'avait pas été envoyée ici par ses très sages électeurs, ce vote aurait peut-être pu passer ${ }^{84}$.

Le sénateur Chafee, qui avait voté contre la motion de M oseley-Braun, déclara :

II ressort de ce débat (...) qu'il existe un groupe dans notre société qui ressent très profondément cette question, et ces opinions ont été très fortement énoncées par le discours extraordinairement excellent de la distinguée sénatrice de I'Illinois. (...) Je dois dire, à regret, que les avis changent rarement dans cette enceinte. Trop souvent, personne ici n'écoute (...). M ais j'ai été persuadé par les positions que j'ai entendues exprimées aujourd'hui, particulièrement par la sénatrice de l'Illinois ${ }^{85}$.

81. I bid., s9263.

82. I bid., s9264.

83. I bid., 59265 .

84. I bid., s9265.

85. Ibid., s9267. 
Le sénateur Bennett de l'Utah déclara que lui, ainsi que d'autres collègues R épublicains, «n'avaient pas conscience des implications plus larges de ce que nous venions de faire» 86 .

A la suite de quelques interventions concernant la procédure parlementaire, une motion fut proposée pour reconsidérer la motion d'ajourner I'amendement. Elle passa par un vote de 76 à 24. Immédiatement après, on tint à nouveau un vote sur la motion de M oseley-Braun d'ajourner l'amendement et la motion passa, 75 à 25.

A près le vote, plusieurs sénateurs ayant voté contre la motion exposèrent les raisons de leur geste. Le sénateur $M$ ack déclara :

Je rejette l'idée que la question devant le Sénat en est une de racisme. Je considère plutôt l'amendement comme ayant été conçu afin de préserver l'héritage de ceux qui descendent fièrement des rangs de ceux qui ont combattu et sont morts au cours de la Guerre civile (...). Ceci n'était pas un vote sur le racisme. C'était un vote dont l'objet était de reconnaître à un groupe de femmes agées le privilège d'honorer leur emblème. J'ai voté pour ce privilège ${ }^{87}$.

M oseley-Braun prit la parole afin de remercier $\mathrm{H}$ eflin de sa bonne grâce ainsi que ceux qui I'avaient soutenue durant le débat. Le sénateur $D$ anforth parla pour s'excuser d'avoir voté en faveur de la motion, disant :

J'en avais presque mal au ventre lorsque j'ai voté, et la raison était que pour prouver d'une manière symbolique mes sentiments de dégoût envers le racisme, j'ai du entaché de racisme des gens innocents qui sont membres d'une organisation (...) à laquelle ils sont fortement attachés (...) et leurs mobiles n'ont rien à voir avec le racisme et rien à voir avec l'approbation de l'esclavage ${ }^{88}$.

II poursuivit en notant que, sur la pelouse de la $\mathrm{M}$ aison Blanche, après la reddition de Lee, Lincoln demanda à l'orchestre de jouer deux chansons : «Yankee D oodle» et «Dixie» : «Je suppose que si le 10 avril 1861 se passait aujourd'hui, nous serions en train de tenir des votes symboliques dans I'enceinte du Sénat des États-U nis pour savoir si l'orchestre aurait dû jouer "Dixie" à cette occasion ${ }^{89}$. Le sénateur Byrd exprima aussi son point de vue que le drapeau confédéré symbolisait le sacrifice des Sudistes pour leur patrie, et non un attachement à l'esclavage. Byrd cita une conversation documentée entre un prisonnier Confédéré et le Yankee qui I'avait capturé; ce dernier demanda au soldat : " "Pourquoi nous combattez-vous ainsi ?" À quoi le soldat confédéré répliqua, "Parce que vous êtes tous ici" ». Byrd poursuivit : "Ce n'était pas du racisme. C e n'était pas la défense de l'esclavage. $C$ 'était un homme protégeant son foyer, sa famille, et son peuple (...). Les Américains d'origine sudiste n'ont pas à défendre l'esclavage afin d'immortaliser l'héritage qui est le leur ${ }^{90}$. II

86. Ibid., s9267.

87. Ibid., s9268.

88 .l bid., 59270.

89. I bid., s9270.

90. Ibid., s9271. 
conclut en fustigeant les Républicains pour avoir changé leur vote, les accusant d'être des «renégats qui avaient couru se mettre à l'abri pour des raisons politiques ${ }^{91}$.

\section{Bibliographie}

Addams, Jane, «The Larger Aspects of the Woman's M ovement», Annals of the A merican Academy of Political and Social Science LVI, 1914, p. 1-8.

Barry, Brian, J ustice as I mpartiality. O xford : O xford University Press, 1995. Benhabib, Seyla, «The Generalized and the Concrete $O$ ther », dans Situating the Self, N ew York : Routledge, 1992.

«Toward a Deliberative M odel of Democratic Legitimacy», dans Seyla Benhabib, dir., D emocracy and D ifference, Princeton : Princeton University Press, 1996, p. 3-19.

Carens, Joseph H, «R ealistic and Idealistic Approaches to the Ethics of Immigration » International M igration R eview 30 (1), 1996, p. 156-70.

Cohen, Joshua, «Deliberation and Democratic Legitimacy », dans $\mathrm{H}$ amlin, Alan et Pettit, Philip, dir., The Good Polity : N ormative A nalysis of the State, Oxford, Basil Blackwell, 1989, p. 17-34.

«Procedure and Substance in Deliberative Democracy », dans

Benhabib, Seyla, dir., D emocracy and D ifference, Princeton : Princeton University Press, 1996, p. 95-119.

Cohen, Joshua et Rogers, Joel, A ssociations and D emocracy, London, Verso, 1995.

Dryzek, John S., D iscursive D emocracy, Cambridge : Cambridge University Press, 1990.

Dworkin, Ronald, «W hat is Equality? Part II : Equality of R esources», Philosophy and Public Affairs 10 (4), 1981, p. 283-345.

Elster, J on, «Strategic U ses of A rgument», dans A rrow, Kenneth, et al, dir., Barriers to Conflict Resolution, N ew York, W.W. Norton, 1995, p. 237249.

Fraser, N ancy. «R ethinking the Public Sphere: A Contribution to the Critique of Actually Existing Democracy », dans Justice Interruptus : Critical Reflections on the "PostSocialist" Condition, N ew York : Routledge, 1997.

Gutmann, Amy et Dennis Thompson, D emocracy and D isagreement, Cambridge, M A. : H arvard University Press, 1996.

$\mathrm{H}$ abermas, Jürgen. « N otes programmatiques pour fonder en raison une éthique de la discussion», M orale et Communication. Conscience

91. La version originale de cet article a été publiée dans Kymlicka, Will et Wayne N orman dir., Citizenship in Diverse Societies, N ew York : Oxford University Press, 2000, pp. 124-152, sous le titre «The Uneasy Alliance of Group Representation and Deliberative Democracy ». II a été traduit de l'anglais par Learry Gagné et D ominique Leydet. 
morale et activité communicationnelle, trad. par Christian Bouchindhomme, Paris : Cerf, 1986, p. 63-134.

"D iscourse Ethics : N otes on a Program of Philosophical Justification », dans M oral Consciousness and Communicative Action, tr. C. Lenhardt et S. W. N icholsen, Cambridge, M A. : M IT Press, 1990, p. 43-115.

Between Facts and Norms : Contributions to a D iscourse Theory of L aw and D emocracy, tr. William Rehg, Cambridge, M A. : M IT Press, 1996.

Johnson, James. «Arguing for Deliberation : Some Skeptical Considerations», dans Elster, J on, dir., D eliberative D emocracy, Cambridge : Cambridge University Press, 1998, p. 161-184.

Kamsler, Victoria, «Democratic Feminism», texte non publié, 1996.

Kymlicka, Will, M ulticultural Citizenship, Oxford : O xford University Press, 1995.

M anin, Bernard, «O n Legitimacy and Deliberation », Political Theory 15 (3), 1987, p. 338-68.

M ansbridge, Jane, «Fighting Power/Using Power» dans Benhabib, Seyla, dir., D emocracy and Difference : Contesting the Boundaries of the Political, Princeton : Princeton University Press, 1996, p. 46-67.

M inow, M artha, M aking All the D ifference, Ithaca : Cornell University Press, 1990.

«From Class Action to M iss Saigon : The Concept of Representation in Law », Cleveland State Law Review 39, p. 269-300.

M oore, M argaret, «On Reasonableness», Journal of Applied Philosophy 13 (2), 1996, p.167-78.

O kin, Susan M oller, Justice, G ender and the Family, N ew York : Basic Books, 1989.

Phillips, Anne, The Politics of Presence, Oxford : Oxford University Press, 1995.

D emocracy \& D ifference, University Park, PA : Pennsylvania State University Press, 1993.

Plessy v. Ferguson, 1896. 163 U.S. 537.

Rawls, J ohn, Libéralisme politique, trad. Catherine Audard, Paris : Presses Universitaires de France, 1995.

A Theory of Justice, Cambridge, M A. : H arvard University Press, 1971.

R ousseau, J.-J ., D u contrat social, Paris : Flammarion, 1966.

Scanlon, T.M . «Contractualism and Utilitarianism», dans Sen, A martya et Williams, Bernard, dir., Utilitarianism and Beyond, Cambridge, Cambridge University Press, 1982, p. 103-128

Sunstein, Cass, «Beyond the Republican Revival », Yale Law Journal 97, 1988, p. 1539-90.

The Partial Constitution, Cambridge, M A., H arvard University Press, 1993. 
Williams, M elissa S., «Justice Toward Groups : Political N ot Juridical », Political Theory 23 (1) :, 1995, p. 67-91. Voice, Trust and M emory : $M$ arginalized G roups and the Failings of Liberal Representation, Princeton : Princeton University Press, 1998.

Young, Iris M arion, Justice and the Politics of Difference, Princeton, N .J., Princeton University Press, 1990. «Justice and Communicative Democracy», dans Gottlieb, R., dir., Tradition, Counter-Tradition, Politics : Dimensions of Radical Democracy, Philadelphia, 1994, p. 123-144. "Communication and the 0 ther : Beyond D eliberative D emocracy », dans Benhabib, Seyla, dir., D emocracy and D ifference, Princeton, N .J. : Princeton University Press, 1996a, p. 120-35. «Difference as a Resource for Democratic Communication», Legal Theory Workshop Series, Faculty of Law, University of Toronto, 22 mars, 1996b. 